TRANSACTIONS OF THE

AMERICAN MATHEMATICAL SOCIETY

Volume 353, Number 5 , Pages 2091-2113

S 0002-9947(01)02712-X

Article electronically published on January 4, 2001

\title{
VARIETIES OF UNISERIAL REPRESENTATIONS IV. KINSHIP TO GEOMETRIC QUOTIENTS
}

\author{
KLAUS BONGARTZ AND BIRGE HUISGEN-ZIMMERMANN
}

\begin{abstract}
Let $\Lambda$ be a finite dimensional algebra over an algebraically closed field, and $\mathbb{S}$ a finite sequence of simple left $\Lambda$-modules. Quasiprojective subvarieties of Grassmannians, distinguished by accessible affine open covers, were introduced by the authors for use in classifying the uniserial representations of $\Lambda$ having sequence $\mathbb{S}$ of consecutive composition factors. Our principal objectives here are threefold: One is to prove these varieties to be 'good approximations' - in a sense to be made precise - to geometric quotients of the (very large) classical affine varieties $\operatorname{Mod}-\operatorname{Uni}(\mathbb{S})$ parametrizing the pertinent uniserial representations, modulo the usual conjugation action of the general linear group. We show that, to some extent, this fills the information gap left open by the frequent non-existence of such quotients. A second goal is that of facilitating the transfer of information among the 'host' varieties into which the considered quasi-projective, respectively affine, uniserial varieties are embedded. For that purpose, a general correspondence is established, between Grassmannian varieties of submodules of a projective module $P$ on one hand, and classical varieties of factor modules of $P$ on the other. Our findings are applied towards the third objective, concerning the existence of geometric quotients. The main results are then exploited in a representation-theoretic context: Among other consequences, they yield a geometric characterization of the algebras of finite uniserial type which supplements existing descriptions, but is cleaner and more readily checkable.
\end{abstract}

\section{INTRODUCTION}

Our purpose is to understand the uniserial representation theory of a finite dimensional algebra $\Lambda$ over an algebraically closed field $K$. This investigation falls into natural parts, namely to describe the classes of uniserial representations sharing a fixed sequence $\mathbb{S}$ of consecutive composition factors. One of the classical molds for the classification problem is provided by the varieties Mod-Uni( $\mathbb{S}$ ) consisting of those points in $\operatorname{Mod}_{\Lambda}^{d}$ which represent uniserial modules with composition sequence $\mathbb{S}$; here $d$ is the number of terms in $\mathbb{S}$, and $\operatorname{Mod}_{\Lambda}^{d}$ is the traditional variety parametrizing all $d$-dimensional $\Lambda$-modules. However, in choosing this mold, one encounters major obstacles on the road towards a classification of the uniserial modules - say, on the basis of quiver and relations of $\Lambda$; namely, the open subvarieties Mod-Uni(S) of $\operatorname{Mod}_{\Lambda}^{d}$ are very large, and even though geometric quotients by the natural $\mathrm{GL}_{d}$-action (the orbits of which are in one-to-one correspondence with

Received by the editors December 8, 1999.

2000 Mathematics Subject Classification. Primary 16G10, 16G20, 16G60, 16P10.

The research of the second author was partially supported by a National Science Foundation grant. 
the isomorphism types of representations) sometimes exist, such optimal situations are far from being readily recognizable. In fact, the representation-theoretic information stored in these varieties is encoded mainly in the $\mathrm{GL}_{d}$-orbits, the geometry of which is notoriously difficult to access from a presentation of $\Lambda$, in general; this is true even though the global varieties $\operatorname{Mod}_{\Lambda}^{d}$ are defined in terms of such presentations. To meet these difficulties, finite collections $V_{\mathbb{S}}$ of smaller affine varieties parametrizing the considered uniserial representations of $\Lambda$ were introduced in [9]. (For purposes of this introduction, we will refer to $V_{\mathbb{S}}$ as though it were a single variety.) These varieties provide a snug fit for the corresponding families of uniserial representations - for more precision see below. Moreover, they have easily computable defining polynomials that stand in a transparent connection to quiver and relations of $\Lambda$, thus providing a viable bridge between quiver-presentations and uniserial representation theory. In [6], the $V_{\mathbb{S}}$ were shown to embed into certain Grassmannians as locally closed subvarieties $\mathcal{G}$-Uni $(\mathbb{S})$; on the other hand, they are isomorphic to certain closed subvarieties of the classical varieties Mod-Uni(S), albeit not to natural ones.

Each of the above settings - Mod-Uni $(\mathbb{S}), V_{\mathbb{S}}$, and $\mathcal{G}-\mathrm{Uni}(\mathbb{S})$ - makes certain aspects of the uniserial representation theory of $\Lambda$ comparatively easy to tackle; first instances of this phenomenon can be found in [6] and [10]. This motivates the wish for tools allowing for smooth transfer of geometric information among the various 'environments' into which the considered uniserial varieties are embedded in particular, such shifts of perspective are desirable as a great deal of general information on the varieties $\operatorname{Mod}_{\Lambda}^{d}$ is available. Machinery for this purpose is provided in Proposition C of Section 2, where we set up a general correspondence between Grassmannian varieties of submodules of a projective module $P$ on one hand and classical varieties of factor modules of $P$ on the other.

Another point on our agenda is to apply this philosophy of reducing technical obstacles through a change of scene to the following problem: Namely, that of understanding the structure and size of the fibres of the representation maps

Considered parametrizing variety $\longrightarrow\{$ isom. types of uniserials with sequence $\mathbb{S}\}$.

While this is a difficult task in the framework of the variety $V_{\mathbb{S}}$, as well as in that of its incarnation inside $\operatorname{Mod}-\operatorname{Uni}(\mathbb{S}) \subseteq \operatorname{Mod}_{\Lambda}^{d}$, it becomes comparatively easy in the Grassmannian setting $\mathcal{G}$-Uni $(\mathbb{S})$. We prove that the fibres of the representation maps are always isomorphic to full affine spaces of small dimension; more precisely, if $\mathbb{S}=(S(0), \ldots, S(l))$, where $S(0)$ is the top simple of the considered uniserial modules, the fibre dimensions are bounded from above by the multiplicity of $S(0)$ in $\mathbb{S}$ diminished by 1 . In addition, the fibres are closed subvarieties of $\mathcal{G}-\operatorname{Uni}(\mathbb{S})$, which, by virtue of Proposition $\mathrm{C}$, says that uniserial representations never have proper uniserial degenerations (parts (2), (3) of Theorem A, Section 2). On learning about our results concerning the fibres, Le Bruyn partially re-proved them in [16], with additional machinery, in the setting of the appropriate Hesselink stratum of the nullcone of representations of a quiver modulo relations. In rough terms, the situation can be summarized by saying that $\mathcal{G}-\mathrm{Uni}(\mathbb{S})$ is always a close approximation to a geometric quotient of $\operatorname{Mod}-\operatorname{Uni}(\mathbb{S})$ modulo the $\mathrm{GL}_{d}$-action, in that the $\mathrm{GL}_{d}$-orbits of Mod-Uni( $\left.\mathbb{S}\right)$ are reduced to comparatively small closed subvarieties of $\mathcal{G}-\operatorname{Uni}(\mathbb{S})$, all of which are geometrically harmless. In other words, the geometric information encoded in the orbits of $\operatorname{Mod}-\operatorname{Uni}(\mathbb{S})$ is shifted to the global geometry of $\mathcal{G}-U n i(\mathbb{S})$. Note however that, in general, geometric quotients of Mod-Uni(S) by 
$\mathrm{GL}_{d}$ fail to exist. Furthermore, the fibre dimension of the representation map

$$
\phi_{\mathbb{S}}: \mathcal{G} \text {-Uni }(\mathbb{S}) \longrightarrow\{\text { isom. types of uniserials with sequence } \mathbb{S}\}
$$

need not be constant on irreducible components (Example 1, Section 3). Since it is generically constant though, it is natural to define the 'uniserial dimension of $\Lambda$ at $\mathbb{S}$ ' as the maximum of the differences 'global dimension minus generic fibre dimension', ranging over the irreducible components of $\mathcal{G}$-Uni $(\mathbb{S})$. As a consequence of the close tie between quiver-presentations of $\Lambda$ and polynomials for $\mathcal{G}$-Uni( $\mathbb{S})-$ see part I of Section 3 - the uniserial dimension of $\Lambda$ at $\mathbb{S}$ is readily available from such a presentation. This yields manageable invariants of $\Lambda$ which play a crucial role in subsequent work on tame algebras [1].

Another of our aims is to decide when $\mathcal{G}$-Uni $(\mathbb{S})$ actually is a geometric quotient of Mod-Uni( $(\mathbb{S})$ by the $\mathrm{GL}_{d}$-action. The answer arises as a consequence of the equivalence of two quotient problems: If $P$ is a projective cover of $S(0)$, the variety $\mathcal{G}$-Uni $(\mathbb{S})$ carries a natural $\operatorname{Aut}_{\Lambda}(P)$-translation action and has a geometric quotient by this action if and only if $\operatorname{Mod}-\operatorname{Uni}(\mathbb{S})$ has a geometric quotient by the $\mathrm{GL}_{d^{-}}$ conjugation; in the positive case, the two quotients are isomorphic (part (1) of Theorem A). The fact that relating geometric quotients to other data on $\Lambda$ is more manageable in the setting of $\mathcal{G}-\mathrm{Uni}(\mathbb{S})$ than for the classical varieties Mod-Uni( $\mathbb{S})$ is again attributable to the fact that the former varieties are closer to such quotients to begin with. Some special cases of our findings along this line overlap with work of King [13]. We note that they also combine with [9, Theorem G] to show that each affine algebraic variety occurs as a geometric quotient Mod-Uni(S)/GL $\mathrm{GL}_{d}$ for suitable choices of $\Lambda$ and $\mathbb{S}$, an indication of the immense complexity of the uniserial representation theory of finite dimensional algebras in general.

As already mentioned, the varieties $\mathcal{G}$-Uni $(\mathbb{S})$ afford concrete insight into the uniserial representation theory, whenever the underlying algebra is given in terms of quiver and relations. One of the focal points of interest has been the problem of characterizing the algebras which have only a finite number of uniserial modules, up to isomorphism, the algebras 'of finite uniserial type' for short; it was posed by Auslander, Reiten, and Smalø in [1, Problems (1) and (2) on p. 411]. Two previous articles addressing it are by the first and second author, respectively. In [4], an inductive characterization of finite uniserial type - based on the Loewy length of the algebra - is given, while [10] provides combinatorial necessary and sufficient conditions in terms of quiver and relations of $\Lambda$, which, however, are separated by a slim gap bridged by a system of linear equations. Two conjectures aiming at a more manageable description were left open in [10; namely, if $\Lambda$ has finite uniserial type, then $(1)$ all of the varieties $\mathcal{G}-\operatorname{Uni}(\mathbb{S})$ are affine spaces, and (2) for each choice of $\mathbb{S}$, there is at most one uniserial module with this sequence of composition factors. The geometric results outlined above enable us to settle the first conjecture in the positive and to thus supplement the confirmation of the second in [4. Moreover, this second conjecture is re-obtained here with a geometric argument. The resulting information, in turn, leads to a new characterization of the algebras of finite uniserial type which is cleaner and, from several viewpoints, more satisfactory than existing ones (see Section 3, part II).

Section 4, finally, contains an alternate proof for the fibre structures of the representation maps, which yields the following interesting fact as a by-product: All endomorphism rings of uniserial $\Lambda$-modules are commutative, a result which, of course, relies heavily on algebraic closedness of the base field. 


\section{Notation AND TOOLS}

Throughout, $\Lambda$ will stand for a finite dimensional algebra over an algebraically closed field $K$, and $J$ will be its Jacobson radical. We assume $\Lambda$ to be basic and may thus suppose that $\Lambda=K \Gamma / I$ is a path algebra modulo relations, with underlying quiver $\Gamma$. It will be convenient to identify the vertices of $\Gamma$ with a full set of primitive idempotents of $\Lambda$; all idempotents to which we will refer will be taken from this set. Moreover, we fix a sequence $\mathbb{S}=(S(0), \ldots, S(l))$ of simples $S(i)=\Lambda e(i) / J e(i)$, where the $e(i)$ are primitive idempotents. The uniserial modules $U$ of composition length $l$ with $J^{i} U / J^{i+1} U \cong S(i)$ we call 'uniserials with composition series $\mathbb{S}$ ' for short, and to the paths of length $l$ passing through the corresponding sequence of vertices $(e(0), \ldots, e(l))$ in that order we will briefly refer as 'paths through $\mathbb{S}$ '. Finally, we call any element $x \in U \backslash J U$ with $e(0) x=x$ a top element of $U$.

The smoothest description of the varieties considered is in the framework of Grassmannians as follows: Setting $m=\operatorname{dim}_{K} \Lambda e(0)-(l+1)$, we denote the Grassmannian of all $m$-dimensional $K$-subspaces of $\Lambda e(0)$ by $\mathcal{G}_{m}(\Lambda e(0))$. Then the quasiprojective subvariety $\mathcal{G}-\operatorname{Uni}(\mathbb{S}) \subseteq \mathcal{G}_{m}(\Lambda e(0))$ consists of those $m$-dimensional submodules $C$ of $\Lambda e(0)$ for which $\Lambda e(0) / C$ is uniserial with composition series $\mathbb{S}$. As we saw in [6], $\mathcal{G}$-Uni( $(\mathbb{S})$ is a locally closed subset of $\mathcal{G}_{m}(\Lambda e(0))$ having a distinguished affine open cover as follows: Given a uniserial module $U$ with composition series $\mathbb{S}$, each path through $\mathbb{S}$ which does not annihilate $U$ is called a mast of $U$. Letting $p$ be a path through $\mathbb{S}$ and $\mathcal{G}-\operatorname{Uni}(p)$ the set of points $C \in \mathcal{G}-\operatorname{Uni}(\mathbb{S})$ such that $\Lambda e(0) / C$ has mast $p$, we obtain a family

$$
(\mathcal{G}-\operatorname{Uni}(p))_{p} \text { a path through } \mathbb{S}
$$

of open affine subsets exhausting $\mathcal{G}$-Uni $(\mathbb{S})$. Clearly, $\mathcal{G}$-Uni $(\mathbb{S})$ comes equipped with a natural surjection $\phi_{\mathbb{S}}$ onto the set of isomorphism classes of uniserial modules with composition series $\mathbb{S}$ which maps a point $C \in \mathcal{G}$-Uni( $\mathbb{S})$ to the class of $\Lambda e(0) / C$. Moreover, it is obvious that the subsets $\mathcal{G}-\operatorname{Uni}(p) \subseteq \mathcal{G}$-Uni $(\mathbb{S})$ are unions of fibres of $\phi_{\mathbb{S}}$. Therefore, we can safely narrow our focus to the affine situation in exploring the fibres. We denote the restriction of the map $\phi_{\mathbb{S}}$ to $\mathcal{G}$-Uni $(p)$ by $\phi_{p}$.

In [6] it was also shown that the affine varieties $\mathcal{G}-\operatorname{Uni}(p)$ have several isomorphic incarnations within alternate settings which frequently offer advantages in solving specific problems. Since, in the sequel, we will move freely back and forth among these varieties, depending on which viewpoint offers an edge, we will briefly recall their definition.

The first of the alternate incarnations, introduced in 9], is combinatorial in nature and requires a bit of terminology for convenient communication of the relevant data. Fix a path $p$ through $\mathbb{S}$, and denote by $p_{0}, \ldots, p_{l}$ its right subpaths; this means that $p=q_{i} p_{i}$ for suitable paths $q_{i}$, our convention being to write $q_{i} p_{i}$ for ' $q_{i}$ after $p_{i}$ '. We assume the paths $p_{i}$ to be ordered in terms of increasing lengths, i.e., length $\left(p_{i}\right)=i$. A path $v \in K \Gamma$ starting in the vertex $e(0)$ is called a route on $p$ if the sequence of vertices through which $v$ successively passes is a subsequence of $(e(0), \ldots, e(l))$. Examples of routes are paths $\alpha p_{m}$ based on 'detours' $\left(\alpha, p_{m}\right)$, as follows: We say that a pair $\left(\alpha, p_{m}\right)$, combining an arrow $\alpha$ with a right subpath $p_{m}$ of $p$, is a detour on $p$ and write $\left(\alpha, p_{m}\right) \varkappa p$, in case $\alpha p_{m}$ fails to be a right subpath of $p$, while there exists a right subpath $p_{s}$ longer than $p_{m}$ which has the same terminal point as $\alpha$. The set of all such indices $s$ is denoted by $I\left(\alpha, p_{m}\right)$. Next we observe that, given any uniserial module $U$ with mast $p$ and top element $x$, we 
have $\alpha p_{m} x=\sum_{i \in I\left(\alpha, p_{m}\right)} k_{i}\left(\alpha, p_{m}\right) p_{i} x$ for unique scalars $k_{i}\left(\alpha, p_{m}\right)$. The prominent role played by detours is due to the obvious fact that it suffices to record the effect of multiplying top elements of $U$ by detours, in order to pin down the isomorphism type of $U$. Reflecting this, the following affine variety $V_{p}$ lives in $\mathbb{A}^{N}$, where $N=\sum_{(\alpha, u) \text { uz }}|I(\alpha, u)|$; it consists of the points $k$ which show up as coordinate strings $\left(k_{i}(\alpha, u)\right)_{i \in I(\alpha, u),(\alpha, u) \text { uz }}$ of uniserials with mast $p$ relative to arbitrary choices of top elements. (For the fact that this set of points in $\mathbb{A}^{N}$ actually is an affine algebraic variety, see [9].) An isomorphism $\psi_{p}: V_{p} \rightarrow \mathcal{G}$-Uni $(p)$ is afforded by the assignment

$$
k=\left(k_{i}(\alpha, u)\right) \mapsto\left(\sum_{(\alpha, u) u p} \Lambda\left(\alpha u-\sum_{i \in I(\alpha, u)} k_{i}(\alpha, u) p_{i}\right)+\sum_{q \text { not a route on } p} \Lambda q e(0)\right)
$$

(see [6]). If $\Phi_{p}$ denotes the surjection from $V_{p}$ onto the set of isomorphism classes of uniserials with mast $p$, which takes $k$ to $\Lambda e(0) / \psi_{p}(k)$, we clearly obtain the following commutative triangle:

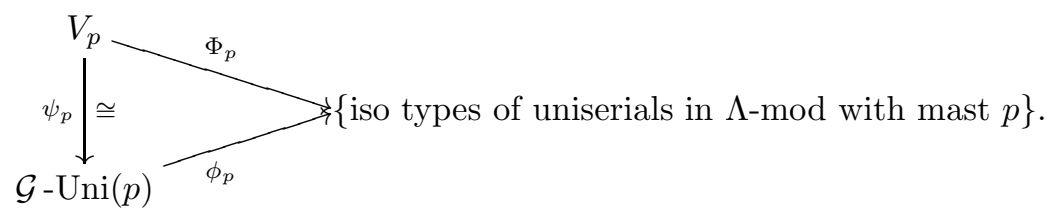

There is another helpful way of looking at the affine varieties $\mathcal{G}-\operatorname{Uni}(p) \cong V_{p}$. Occasionally, it is convenient to identify the affine variety $V_{p}$ (alias $\mathcal{G}-\mathrm{Uni}(p)$ ) with a subvariety, labeled $\operatorname{Uni}(p)$, of the classical variety $\operatorname{Mod}_{\Lambda}^{l+1}$ of bounden $(l+1)$ dimensional representations of $\Lambda$; this identification is justified in [6. Section 3]. Recall that, given a natural number $d$, the variety $\operatorname{Mod}_{\Lambda}^{d}$ of bounden $d$-dimensional representations of $\Lambda=K \Gamma / I$ is defined as follows: If $\Gamma^{*}=$ vertices of $\left.\Gamma\right\} \cup$ \{arrows in $\Gamma$ \}, then $\operatorname{Mod}_{\Lambda}^{d}$ consists of those points $x=(\alpha(x)) \in \prod_{\alpha \in \Gamma^{*}} M_{d}(K)$, the components of which satisfy the relations in $I$. More precisely, $\operatorname{Uni}(p)$ lives inside the open subvariety $\operatorname{Mod}-\operatorname{Uni}(p)$ of $\operatorname{Mod}_{\Lambda}^{l+1}$ containing precisely those points which correspond to uniserial modules with mast $p$. Namely, if $p=\alpha_{l} \alpha_{l-1} \cdots \alpha_{1}$, where the $\alpha_{i}$ are arrows, then $\operatorname{Uni}(p)$ consists of all those points

$$
x=(\alpha(x))_{\alpha \in \Gamma^{*}} \in \operatorname{Mod}-\operatorname{Uni}(p),
$$

for which the $i$ th column of the $(l+1) \times(l+1)$ matrix $\alpha_{i}(x)$ equals the $(i+1)$ st canonical basis vector for $K^{l+1}, 1 \leq i \leq l$; note that the modules corresponding to these points are automatically uniserial with mast $p$. The canonical isomorphism $V_{p} \rightarrow \operatorname{Uni}(p)$ actually identifies the map $\Phi_{p}$ with the restriction of the canonical map $R$ from $\operatorname{Mod}_{\Lambda}^{l+1}$ to the isomorphism types of left $\Lambda$-modules; so in particular, fibres of $\Phi_{p}$ are carried to fibres of $R$ by the mentioned isomorphism of varieties. Note that $\operatorname{Uni}(p)$ is no longer stable under the conjugation action of $\mathrm{GL}_{l+1}$; the closure of $\operatorname{Uni}(p)$ under $\mathrm{GL}_{l+1}$-conjugation coincides with the full subvariety $\operatorname{Mod}-\operatorname{Uni}(p)$ of $\operatorname{Mod}_{\Lambda}^{l+1}$. In fact, the natural group action on $\mathcal{G}-\operatorname{Uni}(p)\left(\cong V_{p} \cong \operatorname{Uni}(p)\right)$, which will provide our main operative tool, does not translate into any conjugation action of a matrix group on Uni $(p)$ in general (see Example 1).

We conclude this sketch of background information with an overview of the mentioned perspectives, including brief descriptions of the relevant varieties. Suppose that $p$ is a path in $\Gamma$ of length $l$. 
- $\mathcal{G}$-Uni $(p)$ is the subvariety of the Grassmannian $\mathcal{G}_{\operatorname{dim}_{K}} \Lambda e(0)-(l+1)(\Lambda e(0))$ consisting of all $\Lambda$-submodules $C \subseteq \Lambda e(0)$ such that $\Lambda e(0) / C$ is uniserial with mast $p$; moreover, $\mathcal{G}-\operatorname{Uni}(\mathbb{S})$ is the union of the $\mathcal{G}$-Uni $(p)$, where $p$ traces all paths through $\mathbb{S}$.

- $V_{p}$ is the set of all coordinate strings glued together from the coordinate vectors of detours inside uniserials with mast $p$.

- $\operatorname{Mod}-\operatorname{Uni}(p)$ is the open subvariety of $\operatorname{Mod}_{\Lambda}^{l+1}$ consisting of the points going with uniserial modules that have mast $p$, and $\operatorname{Mod}-\operatorname{Uni}(\mathbb{S})$ is their union as $p$ again traces the paths through $\mathbb{S}$.

- The Uni $(p)$ are the subvarieties of the corresponding Mod-Uni $(p)$ introduced in the preceding paragraph, and $\operatorname{Uni}(\mathbb{S})$ is their union.

These varieties are related as shown in the following diagram:

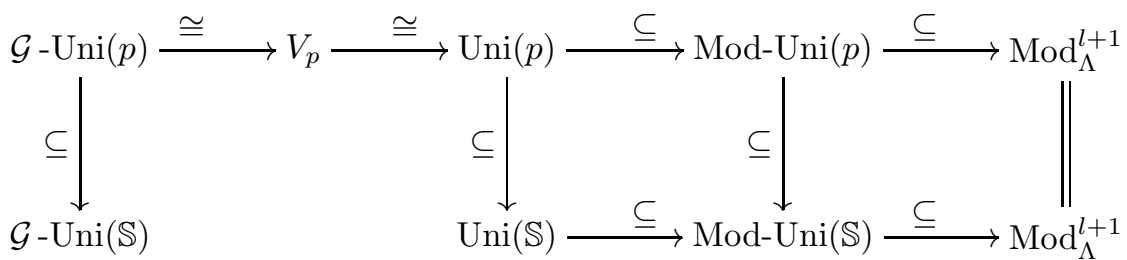

Note that the first row of our diagram consists entirely of affine varieties. As for the second row: On one hand, $\mathcal{G}$-Uni $(\mathbb{S})$ often fails to be affine, while on the other hand, $\operatorname{Uni}(\mathbb{S})$ is a closed subset of the affine variety $\operatorname{Mod}_{\Lambda}^{l+1}$. In particular, this shows that the blank in the second row cannot be filled by an isomorphism in general.

The legitimacy of moving from one incarnation of $\mathcal{G}-\mathrm{Uni}(p)$ to another, as convenience dictates, is guaranteed by the following commutative diagram which ties all of the representation maps together:

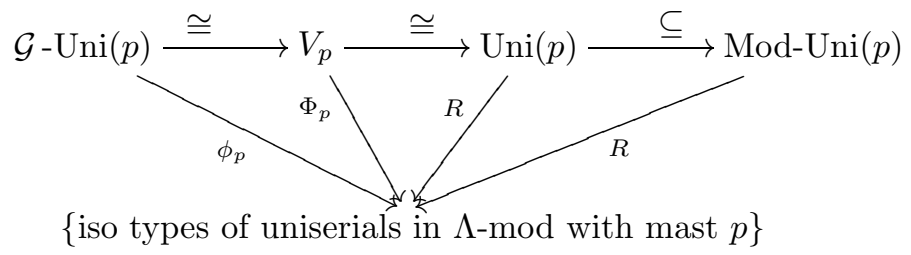

Here $R$ denotes the canonical map from $\operatorname{Mod}_{\Lambda}^{l+1}$ to the set of isomorphism types of $(l+1)$-dimensional left $\Lambda$-modules.

\section{Fibre StRUCtURE AND TRANSFER OF INFORMATION}

Let $G$ be a linear algebraic group acting morphically on a variety $X$. We will use the strongest notion of a quotient of $X$ by $G$, which is as follows: A surjective morphism $\phi: X \rightarrow Y$ of varieties is a geometric quotient of $X$ modulo $G$ if $\phi$ is open, the fibres of $\phi$ coincide with the orbits of $G$, and, for any open subset $U \subseteq Y$, the comorphism $\phi^{0}$ of $\phi$ induces an isomorphism from the ring $\mathcal{O}_{Y}(U)$ of regular functions on $U$ to the subring of $\mathcal{O}_{X}\left(\phi^{-1}(U)\right)$ consisting of those regular functions which are constant on the $G$-orbits of $\phi^{-1}(U)$. Any such geometric quotient is a categorical one, in the sense that each morphism $X \rightarrow Z$ which is constant on the $G$-orbits of $X$ factors uniquely through $\phi$; in particular, geometric quotients are unique up to isomorphism in case of existence. 
Let us focus on a sequence $\mathbb{S}$ of simple left $\Lambda$-modules as before and abbreviate the idempotent $e(0)$ corresponding to the top simple by $e$. We start by introducing a left action of the algebraic group $\operatorname{Aut}_{\Lambda}(\Lambda e)$ on $\mathcal{G}-\operatorname{Uni}(\mathbb{S})$ as follows: Given a point $C \in \mathcal{G}$-Uni( $(\mathbb{S})$, we define $g C$ to be $g(C)$. Since clearly $g$ induces an isomorphism of $\Lambda$-modules $\Lambda e / C \cong \Lambda e / g C$, this yields a well-defined morphic action which leaves the fibres of $\phi_{\mathbb{S}}$ invariant. The action is transitive on the fibres of $\phi_{\mathbb{S}}$ : Indeed, due to the fact that $\Lambda e$ is projective and local, any isomorphism $\Lambda e / C \cong \Lambda e / C^{\prime}$ is induced by an automorphism of $\Lambda e$ taking $C$ to $C^{\prime}$. Moreover, since the isomorphic uniserials $\Lambda e / C$ and $\Lambda e / g(C)$ have the same masts, the affine patches $\mathcal{G}$-Uni $(p)$ are unions of orbits. In other words: For each path $p$ through $\mathbb{S}$, the $\operatorname{Aut}_{\Lambda}(\Lambda e)$-action on $\mathcal{G}-\operatorname{Uni}(\mathbb{S})$ restricts to an action on $\mathcal{G}-\operatorname{Uni}(p)$, the orbits of which coincide with the fibres of the canonical map $\phi_{p}$.

The unipotent group $G$ which will be our mainstay in the sequel is the unipotent radical of $\operatorname{Aut}_{\Lambda}(\Lambda e)$. A typical element of $G$ thus corresponds to right multiplication of $\Lambda e$ by a local unit from the set $\left\{g=e+g^{\prime} \mid g^{\prime} \in e J e\right\}$. Clearly, $\operatorname{Aut}_{\Lambda}(\Lambda e) \cong$ $K^{*} \times G$, and since $K^{*}$ stabilizes all elements of $\mathcal{G}$-Uni $(\mathbb{S})$, the orbits of the $G$-action are still identical with the fibres of the canonical maps. In particular, $G$ stabilizes all of the affine patches $\mathcal{G}-\operatorname{Uni}(p)$.

This essentially yields the second part of our main theorem.

Theorem A. Let $\mathbb{S}=(S(0), \ldots, S(l))$ be a sequence of simple left $\Lambda$-modules, and let $\mu(\mathbb{S})$ denote the multiplicity of $S(0)$ in $\mathbb{S}$.

(1) The classical variety Mod-Uni( $(\mathbb{S})$ has a geometric quotient by the standard $\mathrm{GL}_{d}$-conjugation precisely when $\mathcal{G}-\mathrm{Uni}(\mathbb{S})$ has a geometric quotient by its $\operatorname{Aut}_{\Lambda}(\Lambda e)$ action. In the positive case, the two geometric quotients are isomorphic. Moreover, a geometric quotient of $\mathrm{Mod}-\mathrm{Uni}(\mathbb{S})$ by $\mathrm{GL}_{d}$ exists and coincides with $\mathcal{G}-\mathrm{Uni}(\mathbb{S})$ if and only if $\mu(\mathbb{S})$ equals the $K$-dimension of the endomorphism ring of any uniserial module with composition series $\mathbb{S}$.

(2) There is a morphic action of the unipotent group $G$ on $\mathcal{G}-\mathrm{Uni}(\mathbb{S})$, the orbits of which coincide with the fibres of $\phi_{\mathbb{S}}$. In particular, the fibres are closed subvarieties, and the fibre dimension is generically constant on the irreducible components of $\mathcal{G}$-Uni( $(\mathbb{S})$.

(3) Each fibre of $\phi_{\mathbb{S}}$ is a homogeneous $G$-space under this action. In particular, each fibre is isomorphic to a full affine space. In fact, if $C \in \mathcal{G}-\mathrm{Uni}(\mathbb{S})$, then $G . C=\phi_{\mathbb{S}}^{-1} \phi_{\mathbb{S}}(C) \cong \mathbb{A}^{m(C)}$, where

$$
m(C)=\mu(\mathbb{S})-\operatorname{dim}_{K} \operatorname{End}_{\Lambda}(\Lambda e / C) .
$$

Of course, analogous statements concerning the fibres hold for the canonical surjections $\Phi_{p}$ from the affine varieties $V_{p}$ onto the set of isomorphism types of uniserial modules with mast $p$. It is this rendering which makes the quantities arising in the theorem concretely accessible, an aspect addressed in part I of Section 3.

We will briefly discuss two immediate consequences of Theorem A and then follow with a proof, which will occupy the remainder of this section.

Parts (2) and (3) of Theorem A prompt us to define the uniserial dimension of $\Lambda$ at the sequence $\mathbb{S}$ of simple left $\Lambda$-modules as follows.

Definition. The uniserial dimension of $\Lambda$ at $\mathbb{S}$, denoted uniserdim $\mathbb{S}_{\mathbb{S}} \Lambda$, will be -1 in case $\mathcal{G}-\operatorname{Uni}(\mathbb{S})=\varnothing$; otherwise, uniserdim $\mathbb{S} \Lambda$ will stand for the supremum of the 
following differences: $\operatorname{dim} \mathcal{C}$ minus the generic fibre dimension of $\phi_{\mathbb{S}}$ on $\mathcal{C}$, where $\mathcal{C}$ runs through the irreducible components of $\mathcal{G}$-Uni $(\mathbb{S})$.

The uniserial dimension of $\Lambda$ at $\mathbb{S}$ is an isomorphism invariant of $\Lambda$. Note that it, too, is readily computed by way of the $V_{p}$ : Since the subvarieties $\mathcal{G}-\operatorname{Uni}(p) \cong$ $V_{p}$ form an open cover of $\mathcal{G}-\mathrm{Uni}(\mathbb{S})$, the irreducible components of $\mathcal{G}-\mathrm{Uni}(\mathbb{S})$ are birationally equivalent to those of the $V_{p}$ 's, where $p$ traces the paths through $\mathbb{S}$.

Let us consider the extreme case uniserdim $\mathbb{S} \Lambda=0$. As is to be expected, it occurs precisely when there is a finite positive number of uniserial left $\Lambda$-modules with composition series $\mathbb{S}$ : Indeed, that finiteness of this number implies uniserdim $\mathbb{S} \Lambda \leq 0$ follows directly from the definition. Conversely, if uniserdim $\operatorname{din}_{\mathbb{S}} \Lambda=0$, the number of fibres of $\phi_{\mathbb{S}}$ is indeed finite, for closedness of the fibres in $\mathcal{G}$-Uni $(\mathbb{S})$ guarantees that each irreducible component consists of a single fibre.

As for part (1) of Theorem A: Clearly, the necessary and sufficient condition for $\mathcal{G}-\operatorname{Uni}(\mathbb{S})$ to be a geometric quotient of $\operatorname{Mod}-\operatorname{Uni}(\mathbb{S})$ is satisfied if $\mu(\mathbb{S})=1$, or if $\mu(\mathbb{S})=2$ and $S(0) \cong S(l)$. In general, a presentation of $\Lambda$ in terms of quiver and relations permits us to check whether the condition is satisfied (cf. the computational remarks in Section 3).

It was pointed out to us by W. Crawley-Boevey that the existence of a geometric quotient of $\mathrm{Uni}(\mathbb{S})$ modulo the $\mathrm{GL}_{d}$-action in case $\mu(\mathbb{S})=1$ can also be derived from King's work on moduli spaces [13] as follows: If the quiver $\Gamma$ has $n$ vertices $e_{1}, \ldots, e_{n}$ with $e_{1}=e(0)$, consider the homomorphism $\Theta: \mathrm{K}_{0}(\Lambda$-mod $) \rightarrow \mathbb{Z}$ sending $\left(s_{1}, \ldots, s_{n}\right)$ to $-l s_{1}+\sum_{1<i \leq n} s_{i}$, where $l$ is the length of the sequence $\mathbb{S}=(S(0), \ldots, S(l))$. If $\mu(\mathbb{S})=1$, then clearly all uniserial modules with series $\mathbb{S}$ are $\Theta$-stable and [13, Proposition 5.3] applies.

Combining part (1) of Theorem A with [9, Theorem G], finally, we obtain the following:

Corollary B. Every affine algebraic variety $V$ over $K$ occurs as a geometric quotient of Mod-Uni( $(\mathbb{S})$ by the $\mathrm{GL}_{d}$-action for some finite dimensional algebra and some sequence $\mathbb{S}$ of simple modules. One can even arrange for isomorphisms $V \cong \operatorname{Mod}-\operatorname{Uni}(\mathbb{S}) / \mathrm{GL}_{d} \cong \mathcal{G}-\operatorname{Uni}(\mathbb{S})$.

In rough terms, the proof of part 1 of Theorem A (below) will depart from the following obvious representation-theoretic correspondence: Namely, given a projective module $P$ of $K$-dimension $r$, and natural numbers $m$ and $d$ with $r=m+d$, there is a bijection between the $\operatorname{Aut}_{\Lambda}(P)$-orbits of the $m$-dimensional submodules of $P$ and the isomorphism types of $d$-dimensional factor modules of $P$; it is induced by $U \mapsto P / U$. We will translate this bijection into a correspondence between sets contained in a subvariety of a certain Grassmannian on one hand, and sets contained in a subvariety of $\operatorname{Mod}_{\Lambda}^{d}$ on the other (Proposition C below). The former subvariety is the closed subset $\operatorname{Sub}_{P}^{m}$ of $\mathcal{G}_{m}\left(K^{r}\right)$ consisting of all $m$-dimensional submodules of $P$; here $\mathcal{G}_{m}\left(K^{r}\right)$ denotes the Grassmannian of $m$-dimensional subspaces of $K^{r}$. The subvariety $\operatorname{Fac}_{P}^{d}$ of $\operatorname{Mod}_{\Lambda}^{d}$ which we will consider consists of those points which correspond to $d$-dimensional homomorphic images of $P$. It is an open subvariety of $\operatorname{Mod}_{\Lambda}^{d}$ : Indeed, a $d$-dimensional module $M$ belongs to $\operatorname{Fac}_{P}^{d}$ if and only if the multiplicity of any simple module $S$ in $M / J M$ is smaller than or equal to the multiplicity $c(S)$ of $S$ in $P / \operatorname{rad} P$. But each of these multiplicities is given by the integer-valued, upper semi-continuous function $X \mapsto \operatorname{dim} \operatorname{Hom}_{\Lambda}(X, S)$, and so the set $\operatorname{Fac}_{P}^{d}$, arising as a finite intersection of preimages $(-\infty, c(S)+1)$, is 
indeed open. We observe that $\operatorname{Aut}_{\Lambda}(P)$ naturally acts on $\operatorname{Sub}_{P}^{m}$, while we have the standard conjugation action of $\mathrm{GL}_{d}$ on $\operatorname{Fac}_{P}^{d}$.

In order to relate the projective variety $\operatorname{Sub}_{P}^{m}$ to the quasi-affine variety $\operatorname{Fac}_{P}^{d}$ - or, more precisely, to relate the $\operatorname{Aut}_{\Lambda}(P)$-stable subsets of $\operatorname{Sub}_{P}^{m}$ to the $\mathrm{GL}_{d^{-}}$ stable subsets of $\operatorname{Fac}_{P}^{d}$ - we introduce an intermediary, namely the following closed subvariety $B_{P}^{m, d}$ of $\mathrm{GL}_{r}$ : It contains those matrices in $\mathrm{GL}_{r}$ whose first $m$ columns generate a $\Lambda$-submodule of $P$. In other words, if $p=(\alpha(p))_{\alpha \in \Gamma^{*}}$ is a point in $\operatorname{Mod}_{\Lambda}^{r}$ representing $P$, the spaces generated by the first $m$ columns of the matrices in $B_{P}^{m, d}$ are invariant under left multiplication by all the matrices $\alpha(p)$. Clearly, $\operatorname{Aut}_{\Lambda}(P)$ acts on $B_{P}^{m, d}$. So does the subgroup $\mathcal{H}$ of $\mathrm{GL}_{r}$, consisting of the upper triangular block matrices of the form $\left[\begin{array}{cc}h_{1} & h_{2} \\ 0 & h_{3}\end{array}\right]$, where $h_{1}$ and $h_{3}$ are invertible $m \times m$ and $d \times d$-matrices, respectively. In summary, we obtain a right action of the group $\operatorname{Aut}_{\Lambda}(P) \times \mathcal{H}$ on $B_{P}^{m, d}$, given by $b(f, h)=f^{-1} b h$. Clearly, suitable restriction of the right $\mathcal{H}$-action on $B_{P}^{m, d}$ provides us with a $\mathrm{GL}_{d}$-action on $B_{P}^{m, d}$ as well. Whenever called for, we identify $\mathrm{GL}_{d}$ with its canonical copy inside $\mathcal{H}$.

The next step is to set up a diagram of morphisms

$$
\operatorname{Sub}_{P}^{m} \stackrel{\rho}{\longleftarrow} B_{P}^{m, d} \stackrel{\sigma}{\rightarrow} \operatorname{Fac}_{P}^{d},
$$

where $\rho$ and $\sigma$ are sufficiently well-behaved to afford the transit of geometric information, as we shift subsets from $\operatorname{Sub}_{P}^{m}$ to $\operatorname{Fac}_{P}^{d}$ by applying $\sigma$ to preimages under $\rho$, and vice versa. In particular, we want $\rho$ and $\sigma$ to be equivariant relative to the actions of $\operatorname{Aut}_{\Lambda}(P)$ and $\mathrm{GL}_{d}$, respectively. Moreover, we will construct both maps so as to have local sections, which will at least give us local morphisms between the two varieties flanking $B_{P}^{m, d}$.

Here are the details of the setup: If we let $\rho: B_{P}^{m, d} \rightarrow \operatorname{Sub}_{P}^{m}$ be the map sending a matrix $b \in B_{P}^{m, d}$ to the space generated by its first $m$ columns, then $\rho$ is clearly an $\operatorname{Aut}_{\Lambda}(P)$-equivariant morphism. To define $\sigma: B_{P}^{m, d} \rightarrow \operatorname{Fac}_{P}^{d}$, we start by introducing the map pr: $M_{r}(K) \rightarrow M_{d}(K)$ which assigns to any $r \times r$ matrix its lower right $d \times d$-block. Moreover, we recall that the coordinates $\alpha(p)$ of the point $p$ representing our projective module $P$ are labeled by the elements of $\alpha \in \Gamma^{*}=\left\{e_{1}, \ldots, e_{n}\right\} \cup\{$ arrows in $\Gamma\}$. Noting that, for $b \in B_{P}^{m, d}$ and any $\alpha \in \Gamma^{*}$, the matrix $b^{-1} \alpha(p) b$ is a block matrix of the form

$$
\left(\begin{array}{cc}
* & * \\
0 & \alpha(v)
\end{array}\right)
$$

with $\alpha(v)=\operatorname{pr}\left(b^{-1} \alpha(p) b\right)$, we obtain a point $v=(\alpha(v)) \in \operatorname{Mod}_{\Lambda}^{d}$ which represents $P / \rho(b)$ (by a slight abuse of notation, we identify the point $\rho(b)$ of $\operatorname{Sub}_{P}^{m}$ with the corresponding $\Lambda$-module). Now we define $\sigma$ to be the morphism

$$
b \mapsto\left(\operatorname{pr}\left(b^{-1} \alpha(p) b\right)\right)_{\alpha \in \Gamma^{*}} .
$$

It is easily seen that $\sigma$ is, in fact, a surjective morphism which is equivariant relative to the described operations of $\mathrm{GL}_{d}$ on domain and codomain. Equivariance is just a slice of the good behavior of $\rho$ and $\sigma$, however.

Lemma 1. The morphism $\rho: B_{P}^{m, d} \longrightarrow \operatorname{Sub}_{P}^{m}$ is an $\operatorname{Aut}_{\Lambda}(P)$-equivariant principal right $\mathcal{H}$-bundle. It is the geometric quotient of $B_{P}^{m, d}$ by the action of $\mathcal{H}$.

Proof. Clearly, $\rho$ is the restriction of the $\mathrm{GL}_{r}$-invariant morphism $\tau: \mathrm{GL}_{r} \rightarrow$ $\mathcal{G}_{m}\left(K^{r}\right)$, again assigning to an invertible matrix the space generated by its first 
$m$ columns. Since the canonical right action of $\mathcal{H}$ on $\mathrm{GL}_{r}$ restricts to the $\mathcal{H}$-action on $B_{P}^{m, d}$, it suffices to prove that $\tau$ is a $\mathrm{GL}_{r}$-equivariant principal right $\mathcal{H}$-bundle.

Equivariance being clear, we wish to provide a suitable open covering for $\mathcal{G}_{m}\left(K^{r}\right)$. To that end, let $V$ be any subspace of dimension $d$ of $K^{r}$, and $\mathcal{G}_{V}$ the set of points in $\mathcal{G}_{m}\left(K^{r}\right)$ which complement $V$ in $K^{r}$. The set $\mathcal{G}_{V}$ is an open affine subset of $\mathcal{G}_{m}\left(K^{r}\right)$ : Indeed, choose a basis $b_{m+1}, \ldots, b_{r}$ of $V$, supplement it to a basis $b_{1}, \ldots, b_{r}$ of $K^{r}$, and let $\mathcal{B}$ be the corresponding basis of $\bigwedge^{m}\left(K^{r}\right)$. Then $\mathcal{G}_{V}$ consists precisely of those points which have nonzero $\left(b_{1} \wedge \cdots \wedge b_{m}\right)$-coefficient with respect to $\mathcal{B}$.

To find suitable isomorphisms $\tau^{-1}\left(\mathcal{G}_{V}\right) \rightarrow \mathcal{G}_{V} \times \mathcal{H}$, we observe that, due to the $\mathrm{GL}_{r}$-equivariance of $\tau$, we may restrict our attention to the subspace $V \subseteq K^{r}$ which is generated by the last $d$ canonical basis vectors of $K^{r}$. Then each element $x \in \tau^{-1}\left(\mathcal{G}_{V}\right)$ can be uniquely factored in the form $c \cdot h$ with $h \in \mathcal{H}$ and $c$ a lower triangular matrix of the form $\left[\begin{array}{cc}c_{1} & 0 \\ c_{2} & c_{3}\end{array}\right]$, where $c_{1}, c_{3}$ are the identity matrices of sizes $m \times m$ and $d \times d$, respectively. The assignment $x \mapsto(\tau(x), h)$ yields an isomorphism $\tau^{-1}\left(\mathcal{G}_{V}\right) \rightarrow \mathcal{G}_{V} \times \mathcal{H}$ of varieties, and as $V$ varies, these isomorphisms satisfy the required compatibility conditions.

In particular, the morphism $\rho$ has local sections, and hence the final statement now follows from [5, Lemma 5.5].

There is an alternate guise of the $\mathrm{GL}_{d}$-conjugation on $\operatorname{Fac}_{P}^{d}$ which will be useful in the proof of Lemma 2; namely, if we define a right action of $\operatorname{Aut}_{\Lambda}(P) \times \mathcal{H}$ on $\operatorname{Fac}_{P}^{d}$ by $v(f, h)=(\operatorname{pr} h)^{-1} v(\operatorname{pr} h)$, then the morphism $\sigma$ becomes $\operatorname{Aut}_{\Lambda}(P) \times \mathcal{H}$ equivariant, since the fibres of $\sigma$ are stable under $\operatorname{Aut}_{\Lambda}(P)$. Noting that the map pr $: M_{r}(K) \rightarrow M_{d}(K)$ restricts to a group homomorphism pr $\left.\right|_{\mathcal{H}}: \mathcal{H} \rightarrow \mathrm{GL}_{d}$, and denoting its kernel by $N$, we obtain

Lemma 2. The morphism $\sigma: B_{P}^{m, d} \rightarrow \operatorname{Fac}_{P}^{d}$ is $\mathrm{GL}_{d}$-equivariant and smooth. It is the geometric quotient of $B_{P}^{m, d}$ by the $\operatorname{Aut}_{\Lambda}(P) \times N$-action.

Proof. For our analysis of $\sigma$ it will be helpful to 'expand' the picture and look at the following isomorphic copy $Z$ of the variety $B_{P}^{m, d}$; it is located as a locally closed subvariety inside the product

$$
\operatorname{Fac}_{P}^{d} \times K^{d \times r} \times B_{P}^{m, d},
$$

namely, as the image under the morphism $\zeta$ sending any point $b \in B_{P}^{m, d}$ to $(\sigma(b)$, $\left.[0 E] b^{-1}, b\right)$, where $E$ is the $d \times d$ identity matrix. One checks that the map $\psi=$ $[0 E] b^{-1}$ satisfies $\psi \alpha(p)=\alpha(\sigma(b))$ for $\alpha \in \Gamma^{*}$ and thus concludes that $\psi$ is a $\Lambda$ epimorphism from $P$ to $P / \rho(b)$. Clearly, $\zeta: B_{P}^{m, d} \rightarrow Z=\operatorname{Im}(\zeta)$ is a bijective morphism, the inverse of which is just the projection onto the third component, whence $\zeta$ is an isomorphism. The map $\zeta$ is even an $\operatorname{Aut}_{\Lambda}(P) \times \mathcal{H}$-equivariant isomorphism if one equips $Z$ with the right $\operatorname{Aut}_{\Lambda}(P) \times \mathcal{H}$-action given by

$$
\left(\sigma(b),[0 E] b^{-1}, b\right) \cdot(h, f)=\left((\operatorname{pr} h)^{-1} \sigma(b)(\operatorname{pr} h),[0 E](b h)^{-1} f, f^{-1} b h\right)
$$

for $b \in B_{P}^{m, d}, f \in \operatorname{Aut}_{\Lambda}(P)$ and $h \in \mathcal{H}$. In verifying our assertions, we are hence free to replace $\sigma$ by the projection $\pi: Z \rightarrow \operatorname{Fac}_{P}^{d}$ onto the first component.

First we check that the fibres of $\pi$ coincide with the $\operatorname{Aut}_{\Lambda}(P) \times N$-orbits of $Z$ under the specified action. The group $N$ being equal to

$$
\left\{\left[\begin{array}{cc}
h_{1} & h_{2} \\
0 & E
\end{array}\right] \mid h_{1} \in \mathrm{GL}_{m}, h_{2} \in K^{m \times d}\right\},
$$


it is clear that $\sigma$, and hence also $\pi$, is constant on these orbits. Conversely, consider two points in $Z$ with the same image under $\pi$, say $(v, \psi, b)$ and $\left(v, \psi^{\prime}, b^{\prime}\right)$. Then $\psi$ and $\psi^{\prime}$ both represent epimorphisms $P \rightarrow P / \rho(b)$, where the factor module $P / \rho(b)$ represents the point $v \in \mathrm{Fac}_{P}^{d}$, and by factoring each of the two maps into a projective cover of $P / U$ and a trivial component, we see that $\psi^{\prime}=\psi f$ for a suitable $\Lambda$-automorphism $f$ of $P$; in other words, $\psi^{\prime}$ belongs to the $\operatorname{Aut}_{\Lambda}(P)$-orbit of $\psi$, and it is harmless to assume $\psi=\psi^{\prime}$. But this implies that $\left(b^{\prime}\right)^{-1}=h b^{-1}$ for a suitable element $h \in N$ and thus forces $b^{\prime}$ into the $N$-orbit of $b$.

For the first assertion of the lemma, it now suffices to show that $\pi$ has local sections (see [5, Lemma 5.5]). We prove this in tandem with smoothness, by factoring $\pi$ in the form $\pi=\pi^{\prime \prime} \pi^{\prime}$, the first map being the projection

$$
\pi^{\prime}: Z \rightarrow X, \quad(v, \psi, b) \mapsto(v, \psi)
$$

and the second

$$
\pi^{\prime \prime}: X \rightarrow \operatorname{Fac}_{P}^{d}, \quad(v, \psi) \mapsto v ;
$$

here $X$ is the variety consisting of all pairs $(v, \psi) \in \operatorname{Fac}_{P}^{d} \times K^{d \times r}$ such that $\operatorname{rank}(\psi)$ $=d$ and $\psi \alpha(p)=\alpha(v) \psi$ for $\alpha \in \Gamma^{*}$. In other words, $\psi$ is a $\Lambda$-epimorphism $P \rightarrow P / U$, where $P / U \in \Lambda$-mod corresponds to $v$.

To see that $\pi^{\prime}$ has local sections, let $(v, \psi)$ be a point in $X$, and write $\psi=$ $\left(\psi_{1}, \psi_{2}\right)$, where $\psi_{1} \in K^{d \times m}$ and $\psi_{2} \in K^{d \times d}=M_{d}(K)$. Since $\operatorname{rank}(\psi)=d$, we can find $g \in \mathrm{GL}_{r}$ such that $(\psi g)_{2} \in \mathrm{GL}_{d}$. Pick an open neighborhood $\mathcal{N}$ of $(v, \psi)$ in $X$, with the property that $\left(\psi^{\prime} g\right)_{2} \in \mathrm{GL}_{d}$ for all points $\left(v^{\prime}, \psi^{\prime}\right) \in \mathcal{N}$, and check that the morphism $\mathcal{N} \rightarrow Z$, given by $\left(v^{\prime}, \psi^{\prime}\right) \mapsto\left(v^{\prime}, \psi^{\prime}, s\left(v^{\prime}, \psi^{\prime}\right)\right)$, with

$$
s\left(v^{\prime}, \psi^{\prime}\right)=g\left[\begin{array}{cc}
E_{m} & 0 \\
-\left(\psi^{\prime} g\right)_{2}^{-1}\left(\psi^{\prime} g\right)_{1} & \left(\psi^{\prime} g\right)_{2}
\end{array}\right]
$$

is a section of $\pi^{\prime}$ over $\mathcal{N}$. Since all of the maps $\psi^{\prime}$ are surjective, Lemma 3.4 of [5], and an obvious 'affine shift' thereof, yields the following two bundles $Z_{0}$ and $Z_{1}$ with base $\mathcal{N}$ : Namely, the vector bundle $Z_{1}$ consisting of all triples $\left(v^{\prime}, \psi^{\prime}, c\right)$ with $\left(v^{\prime}, \psi^{\prime}\right) \in \mathcal{N}$ and $c \in M_{r}(K)$ satisfying $\psi^{\prime} c=0$, and the affine bundle $Z_{0}$ consisting of the triples $\left(v^{\prime}, \psi^{\prime}, b\right)$ with $\left(v^{\prime}, \psi^{\prime}\right) \in \mathcal{N}$ and $b \in M_{r}(K)$ satisfying $\psi^{\prime} b=\left[\begin{array}{ll}0 & E\end{array}\right]$. We note that $Z_{0}$ contains $Z$ as an open subset: Indeed, given $\left(v^{\prime}, \psi^{\prime}, b\right) \in Z_{0}$, we have $b \in B_{P}^{m, d}$ if and only if $b \in \mathrm{GL}_{r}$, since the equality $\psi^{\prime} b=\left[\begin{array}{ll}0 & E\end{array}\right]$ forces the first $m$ columns of $b$ to span the kernel of the $\Lambda$-homomorphism $\psi^{\prime}$. Moreover, we observe that $Z_{1}$ and $Z_{0}$ are isomorphic bundles via the assignment $\left(v^{\prime}, \psi^{\prime}, c\right) \mapsto$ $\left(v^{\prime}, \psi^{\prime}, c+s\left(v^{\prime}, \psi^{\prime}\right)\right)$. Hence $\pi^{\prime}$ is smooth, being the restriction of the vector bundle projection $Y \rightarrow \mathcal{N}$ to the open subvariety $Z$ of $Z_{0}$.

To prove that $\pi^{\prime \prime}$ is also smooth and equipped with local sections, we consider the following extension $\pi_{0}^{\prime \prime}$ of $\pi^{\prime \prime}$ to the variety $X_{0}$ of all pairs $(v, \psi) \in \operatorname{Fac}_{P}^{d} \times K^{d \times r}$ satisfying $\psi \alpha(p)=\alpha(v) \psi$ for $\alpha \in \Gamma^{*}$; in other words, the second components of the pairs we single out run through the $\Lambda$-homomorphisms $P \rightarrow P / \rho(v)$. Let $\pi_{0}^{\prime}$ be the projection $X_{0} \rightarrow \operatorname{Fac}_{P}^{d}$ onto the first component. Since $X$ is contained in $X_{0}$ as an open subset and $\pi^{\prime \prime}$ is the restriction of $\pi_{0}^{\prime}$ to $X$, we need only establish our claim for $\pi_{0}^{\prime}$, and to do so, it suffices to check that the latter map is a vector bundle projection. But this follows again from [5, Lemma 3.4], in view of the following two observations: (a) the set of $\Lambda$-homomorphisms $P \rightarrow P / \rho(v)$ inside $K^{d \times r}$ arises as the solution set of a homogeneous system of linear equations, the coefficient matrix $A(v)$ of which depends morphically on $v$ - recall that $P$ is fixed; and (b), 
the rank of $A(v)$ is locally constant on $\operatorname{Fac}_{P}^{d}$. Indeed the nullity of $A(v)$ equals $\operatorname{dim}_{K} \operatorname{Hom}_{\Lambda}(P, P / \rho(v))$. Thus local constancy can be gleaned from the fact that $\operatorname{dim}_{K} \operatorname{Hom}_{\Lambda}(P,-)$ is constant on the intersections of $\operatorname{Fac}_{P}^{d}$ with the open connected components of $\operatorname{Mod}_{\Lambda}^{d}$, because each such component consists of modules having the same class in the Grothendieck group $\mathrm{K}_{0}(\Lambda$-mod).

Proposition C. There is an inclusion-preserving one-to-one correspondence between the $\operatorname{Aut}_{\Lambda}(P)$-stable subsets of $\mathrm{Sub}_{P}^{m}$ and the $\mathrm{GL}_{d}$-stable subsets of $\mathrm{Fac}_{P}^{d}$ given by $M \mapsto \sigma \rho^{-1}(M)$. Both ways, this correspondence preserves openness, closures, connected and irreducible components, as well as types of singularities. Furthermore, an $\operatorname{Aut}_{\Lambda}(P)$-stable subvariety $M$ of $\operatorname{Sub}_{P}^{m}$ admits a geometric quotient by $\operatorname{Aut}_{\Lambda}(P)$ if and only if the corresponding $\mathrm{GL}_{d}$-stable subvariety of $\mathrm{Fac}_{P}^{d}$ admits a geometric quotient by the $\mathrm{GL}_{d}$-action; in the positive case, both of these quotients are isomorphic to the geometric quotient of $\rho^{-1}(M)$ by $\operatorname{Aut}_{\Lambda} P \times \mathcal{H}$.

Proof. All of the statements except for the last are straighforward consequences of Lemmas 1, 2 and the general background given in [5, Section 5]. For the final assertion, we use the fact that $\rho$ and $\sigma$ are flat morphisms and apply [5, Lemma $5.9]$.

We require one more ingredient for the proof of Theorem A. Recall that, given any closed subgroup $H$ of an algebraic group $G$, the set $G / H$ of left cosets of $H$ in $G$ can be equipped with a structure of quasiprojective variety which makes the canonical surjection $G \rightarrow G / H$ a geometric quotient of $G$ relative to the action of $H$ by right translation (see, e.g., [7, Theorem 6.8]). Note that $G / H$ carries a canonical transitive left $G$-action. We will call a $G$-space $X$ a homogeneous $G$-space in case there exists a $G$-equivariant isomorphism $X \rightarrow G / H$ for a suitable closed subgroup $H$ of $G$. As is well-known (see 7, Prop. 6.7]), provided that the action of $G$ on $X$ is transitive, the orbit map $G \rightarrow X, g \mapsto g x_{0}$ for $x_{0} \in X$, induces an isomorphism $G / \operatorname{Stab}\left(x_{0}\right) \rightarrow X$ if and only if this orbit map is separable. In case $K$ has characteristic zero, separability is automatic.

The following structure theorem for homogeneous spaces of unipotent groups is a consequence of a theorem of Rosenlicht [17, Theorem 1].

Theorem (Rosenlicht). Let $G$ be a unipotent algebraic group. Then any homogeneous $G$-space is isomorphic to a full affine space $\mathbb{A}^{r}$.

Proof of Theorem A. We postpone our argument for part (1) until the end of the proof.

Part (2). The second assertion under (2) is well-known to follow from the first: Use [15, II.2.6] to deduce the generic behavior of the fibre dimension. To obtain closedness of the $G$-orbits, we need only show that their intersections with the patches $\mathcal{G}-\mathrm{Uni}(p)$ of our affine cover of $\mathcal{G}-\mathrm{Uni}(\mathbb{S})$ are all closed. But since each of the $\mathcal{G}-\operatorname{Uni}(p)$ is stabilized by $G$ - see the discussion preceding Theorem $\mathrm{A}$ - this is guaranteed by Kostant's result that the orbits of a unipotent group acting on an affine variety are closed (cf. [12, p. 115, Exercise 8]).

Part (3). Fix $C \in \mathcal{G}$-Uni(S). In view of Rosenlicht's theorem, the claimed structure of the fibre $\mathcal{F}=G$.C will follow if we can show $\mathcal{F}$ to be a homogeneous $G$-space. It thus suffices to verify that the orbit map $G \rightarrow \mathcal{F}, g \mapsto g C$, is separable. To this end, it is enough to check that the fibre $G_{1}$ of the orbit map is reduced (combine [7, Prop. 6.7] with [15, AI.5.5, Satz 2] to see this). Now reducedness of 
the stabilizer subgroup $G_{1}$ of $C$ is in turn automatic if $G_{1}$ arises as the solution set of a system of linear equations over $K$ (for background on reduced fibres see [15] AI.2.5, 2.6]). In our situation, the latter condition can be verified as follows.

Suppose that the uniserial module $\Lambda e / C$ has mast $p$, and let $p_{0}, \ldots, p_{l}$ be the right subpaths as before. We know that there is a unique family of scalars $k_{i}(\alpha, u)$ such that $\alpha u-\sum_{i \in I(\alpha, u)} k_{i}(\alpha, u) p_{i} \in C$. Since, as a $\Lambda$-module, $C$ is generated by these differences and the non-routes on $p$, the condition $g C=C$ is clearly equivalent to the requirement that

$$
\text { all of the } g \text {-shifts } g \cdot\left(\alpha u-\sum_{i \in I(\alpha, u)} k_{i}(\alpha, u) p_{i}\right) \text { for }(\alpha, u) \varkappa p \text { belong to } C \text {. }
$$

Let $B$ be a $K$-basis for $e J e$, i.e., $G^{\text {op }}=\left\{e+\sum_{b \in B} l_{b} b \mid l_{b} \in K\right\}$. Using the fact that the images of the $p_{i}$ form a basis for $\Lambda e / C$, we expand the elements $\alpha u b$ and $p_{i} b$ for $b \in B$ modulo $C$. A comparison of coefficients of the $p_{i}+C$ then shows condition $(\dagger)$ to be tantamount to a linear system of equations for the coefficients $l_{b}$ of $g$.

To compute the dimension of the fibre G.C, observe that we have a group epimorphism $\chi: G_{1} \rightarrow \operatorname{Aut}_{\Lambda}^{u}(\Lambda e / C)$, where $\chi_{g}(\lambda+C)=g \lambda+C$. Denoting by $G_{0} \triangleleft G_{1}$ the

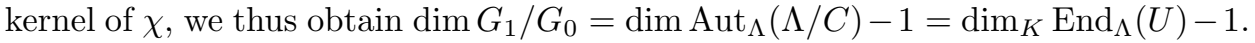
It thus suffices to prove that $\operatorname{dim} G / G_{0}=\mu(\mathbb{S})-1$.

Abbreviating this last difference by $t$, we find precisely $t$ distinct oriented cycles of positive length among the right subpaths $p_{1}, \ldots, p_{l}$ of $p$; let these be $w_{1}, \ldots, w_{t}$. Moreover, let $x$ be the residue class $e+C$, and consider the following set of top elements of $\Lambda e / C$, namely

$$
\mathcal{T}=\left\{\left(e+\sum_{i=1}^{t} c_{i} w_{i}\right)+C \mid\left(c_{1}, \ldots, c_{t}\right) \in \mathbb{A}^{t}\right\} .
$$

Clearly, $G$ acts transitively on $\mathcal{T}$, and $G_{0}$ is precisely the stabilizer subgroup of $e+C$. This gives us $\operatorname{dim} G / G_{0}=t$ as desired and completes the proof of the theorem.

Part (1). We will apply Proposition $\mathrm{C}$ to the situation $d=l+1$, the projective module $P=\Lambda e$, where $e$ is the vertex going with the top simple of $\mathbb{S}$, and the subvariety $M=\mathcal{G}-\mathrm{Uni}(\mathbb{S}) \subseteq \operatorname{Sub}_{P}^{m}$. Under the bijection of Proposition $\mathrm{C}$, the variety $M$ is paired with the $\mathrm{GL}_{d}$-stable open subvariety Mod-Uni( $\left.\mathbb{S}\right)$ of $\operatorname{Fac}_{P}^{d}$, and consequently the first two claims under (1) follow immediately from the proposition. Concerning the final assertion, we just need to know when $\operatorname{Aut}_{\Lambda}(\Lambda e)$ acts trivially on $\mathcal{G}$-Uni( $(\mathbb{S})$. But, keeping in mind that the orbits of the $\operatorname{Aut}_{\Lambda}(\Lambda e)$-action coincide with those of the $G$-action, we obtain the answer from part (3) of Theorem A: This happens if and only if $\mu(\mathbb{S})-\operatorname{dim}_{K} \operatorname{End}_{\Lambda} U=0$ for all uniserial modules $U$ with composition series $\mathbb{S}$.

Remarks. 1. In view of Theorem A, Proposition $\mathrm{C}$ tells us that the $\mathrm{GL}_{d}$-orbits of $\operatorname{Mod}-U n i(\mathbb{S})$ are closed in Mod-Uni( $\mathbb{S})$; in other words, that uniserial modules have no proper uniserial degenerations, and we thus re-encounter Proposition E of [6].

2. As we pointed out earlier, our proof for the first part of Theorem A is derived from a geometric re-interpretation of an obvious module-theoretic correspondence: namely, that between the $\operatorname{Aut}_{\Lambda}(P)$-orbits of submodules of a fixed dimension $m$ inside a projective module $P$ and the isomorphism types of factor modules of dimension $\operatorname{dim} P-m$ of $P$. There are other time-honored ways of studying modules 
which translate into similar geometric pictures. For instance, if one focuses on the maps $P_{1} \rightarrow P_{2}$ of fixed rank between two projective modules $P_{1}$ and $P_{2}$, then the cokernels of two maps $f$ and $g$ are isomorphic if and only if $f$ and $g$ are conjugate under the obvious $\operatorname{Aut}\left(P_{1}\right) \times \operatorname{Aut}\left(P_{2}\right)$-action on $\operatorname{Hom}\left(P_{1}, P_{2}\right)$. To obtain the corresponding result at the geometric level, one can again use some canonical bundle constructions modeling our approach to Proposition C. Analogous correspondences arise if one interprets a module as the image of a map from a projective to an injective object or as the kernel of a map between two injectives. We leave the details of the ensuing geometric setups to the reader.

3. Implicitly, part of the relationship between Grassmannians and module varieties developed above already played a role in Gabriel's article 'Finite representation type is open'; see [8]. To elaborate the connection a bit, we denote by $\mathrm{Alg}_{s}$ the affine variety of $s$-dimensional associative $K$-algebras with identity, by $\operatorname{Alg}_{s} \operatorname{Mod}^{r}$ the variety of all pairs $(a, v)$ composed of a point $a$ of $\operatorname{Alg}_{s}$ and an $r$-dimensional $a$-module $v$, and by $\operatorname{Alg}_{s} \mathrm{Sub}^{m}$ the variety of pairs $(a, u)$, where $m=(s-1) r$ and the second component is an $m$-dimensional submodule $u$ of the $r$ th power of the regular representation of $a$; somewhat sloppily we denote the latter by $a^{r}$. Finally, $B^{m, r}$ will stand for the variety of pairs $(a, b)$, consisting of $a \in \mathrm{Alg}_{s}$ and matrices $b$ running through the elements of $\mathrm{GL}_{r s}$ with the property that the first $m$ columns span a submodule of $a^{r}$. This setup comes with morphisms $\rho: B^{m, r} \rightarrow \operatorname{Alg}_{s} \mathrm{Sub}^{m}$ and $\sigma: B^{m, r} \rightarrow \operatorname{Alg}_{s} \operatorname{Mod}^{r}$ defined as before. They can be supplemented to a commutative diagram

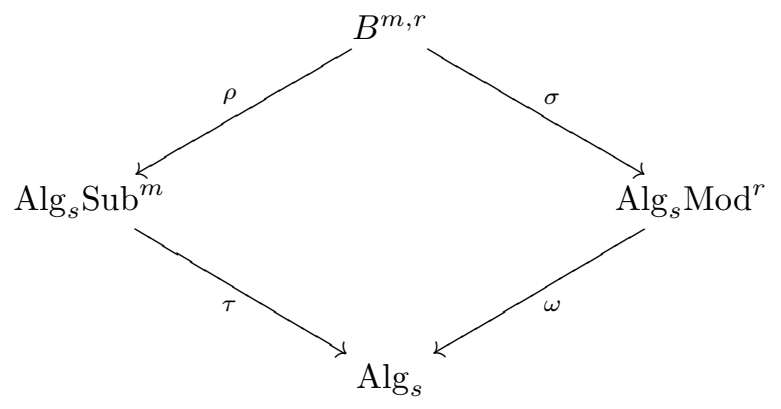

where $\tau$ and $\omega$ are the obvious projections. Again $\rho$ and $\sigma$ are smooth morphisms, both equivariant with respect to the appropriate group actions; moreover, $\tau$ is proper. A crucial difference, compared with the setup of Proposition $\mathrm{C}$, however, lies in the fact that the group $\operatorname{Aut}(P)$ needs to be replaced by the obvious smooth group scheme over $\mathrm{Alg}_{s}$ consisting of automorphisms of $a^{r}$.

One of the key points proved in Gabriel's paper is the fact that $\omega$ maps any $\mathrm{GL}_{r}$-stable closed subset $X$ of $\operatorname{Alg}_{s} \operatorname{Mod}^{r}$ to a closed subset $Y$ of $\operatorname{Alg}_{s}$. This can be gleaned from our picture, because $Y=\tau \rho \sigma^{-1}(X)$ : Indeed, $\rho$ is a principal $\mathcal{H}$ bundle, where $\mathcal{H}$ denotes the subgroup of $\mathrm{GL}_{r s}$ relevant to the present situation, and consequently $\rho$ maps the closed $\mathcal{H}$-stable subset $\sigma^{-1}(X)$ of $\operatorname{Alg}_{s} \operatorname{Mod}^{r}$ to a closed subset of $\operatorname{Alg}_{s} \mathrm{Sub}^{m}$; the proper morphism $\tau$ then takes $\rho \sigma^{-1}(X)$ in turn to a closed set. Gabriel's original argument is essentially this, but does not specify the 'intermediate' variety $B^{m, r}$. However, Kraft introduced an auxiliary variety similar to ours in his treatment of Gabriel's theorem in [14]. 


\section{Applichtions of Theorem A}

(I) Computational remarks and an example. We begin with remarks indicating how the quantities appearing in Theorem A, such as the dimension of the fibre above a class $\Phi_{p}(k)$ of uniserial modules, can be determined algorithmically from quiver and relations of $\Lambda$. Subsequently, we will give an example that serves a twofold purpose: Namely, (a) it shows that the fibre dimension can assume multiple values on a given irreducible component of $\mathcal{G}-\mathrm{Uni}(\mathbb{S})$, and $(\mathrm{b})$ it displays constraints which, in general, render it impossible to translate the described $G$-action on the variety $\mathcal{G}-\mathrm{Uni}(\mathbb{S})$ into a more 'traditional' conjugation action of a unipotent matrix group on the affine variety $\operatorname{Uni}(\mathbb{S})=\bigcup \operatorname{Uni}(p) \subseteq \operatorname{Mod}_{\Lambda}^{l+1}$, where $p$ traces the paths through $\mathbb{S}$. Such a translation is not even possible for the individual affine patches $\mathcal{G}-\operatorname{Uni}(p)$ and $\operatorname{Uni}(p)$, respectively (keep in mind that they are unions of orbits, so that our $G$-action on $\mathcal{G}-\mathrm{Uni}(\mathbb{S})$ restricts to actions on the $\mathcal{G}$-Uni $(p))$. More precisely, while according to Section $1, \mathcal{G}-\operatorname{Uni}(p)$ and the closed subvariety $\operatorname{Uni}(p)$ of $\operatorname{Mod}_{\Lambda}^{l+1}$ are always isomorphic, in general there is no isomorphism $\mathcal{G}$-Uni $(p) \rightarrow \operatorname{Uni}(p)$ which reduces the $G$-action on $\mathcal{G}$-Uni $(p)$, via some group homomorphism $G \rightarrow \mathrm{GL}_{l+1}$, to the conjugation action of a unipotent subgroup $H \leq \mathrm{GL}_{l+1}$ on $\operatorname{Uni}(p)$.

1. First we give a compact review of a method to determine defining polynomials for the affine varieties $V_{p} \cong \mathcal{G}$-Uni $(p)$ from quiver and relations of $\Lambda$; for more detail we refer to 9 .

Let $\Lambda=K \Gamma / I$ and $p=p e$ a path in $\Gamma$. Consider the noncommutative polynomial ring $K \Gamma\left[X_{i}(\alpha, u) \mid(\alpha, u)\right.$ «p, $\left.i \in I(\alpha, u)\right]$; here, the variables $X_{i}(\alpha, u)$ commute with the coefficients in $K \Gamma$ and with each other. We introduce a congruence relation relative to addition and left multiplication on this ring, denoted by '스' and generated by the following substitution equations, namely, the congruences

$$
\alpha u \widehat{=} \sum_{i \in I(\alpha, u)} X_{i}(\alpha, u) v_{i}(\alpha, u) \quad \text { and } \quad q \widehat{=} 0
$$

for $(\alpha, u) \Downarrow p$ and for those paths $q$ which fail to be routes on $p$. Given any element $z \in K \Gamma$, there are unique elements $\tau_{i}(z)$ in the commutative polynomial ring $K\left[X_{i}(\alpha, u) \mid(\alpha, u) \gtrless p, i \in I(\alpha, u)\right]$ with the property that

$$
z \widehat{=} \sum_{i=0}^{l} \tau_{i}(z) p_{i} .
$$

Provided one starts with a set of generators for the left ideal $I e$ of $K \Gamma$, say $z_{1}, \ldots, z_{r}$, the corresponding polynomials $\tau_{i}\left(z_{j}\right), 0 \leq i \leq l, 1 \leq j \leq r$, determine $V_{p}$; namely, $V_{p}=V\left(\tau_{i}\left(z_{j}\right) \mid i, j\right)$. In practice, these polynomials are obtained by starting with a choice of $z_{j}$ and successively inserting the substitution equations into monomials of the form $f(X) v$, where $f(X) \in K[X]$ and $v \in K \Gamma$ is a path (for more detail, see [9]).

2. Next we show how to obtain the fibre dimensions of the maps $\Phi_{p}$ - or, equivalently, of the maps $\phi_{\mathbb{S}}$. In view of Theorem $A$, this boils down to determining the rank of a certain $t \times t$-matrix $A(k)$, where $t=\mu(\mathbb{S})-1$.

Given any point $k \in V_{p}$, the dimension $\delta(k)=\operatorname{dim}_{K} \operatorname{End}_{\Lambda}(U)$, where $U$ is any representative of the isomorphism class $\Phi_{p}(k)$, can be computed along the following line. (Actually, our instructions can be used to explicitly calculate $\operatorname{Aut}_{\Lambda}^{u}(U)$.) Suppose $p$ passes through the sequence $\mathbb{S}$ with top simple $S(0)=\Lambda e / J e$, and let $w_{0}=e, w_{1}, \ldots, w_{t}$ be the different right subpaths of $p$ ending in the starting vertex 
$e$; clearly, $\mu(\mathbb{S})=t+1$ by the definition of $\mu$. For a suitably chosen top element $x=e x$ of $U$, we then have

$$
\alpha u x=\sum_{i \in I(\alpha, u)} k_{i}(\alpha, u) v_{i}(\alpha, u) x
$$

for all $(\alpha, u) \varkappa p$. We indicate how to set up a system of linear equations for those $t$-tuples $\left(c_{1}, \ldots, c_{t}\right)$ of scalars for which the assignment

$$
x \mapsto x+\sum_{i=1}^{t} c_{i} w_{i} x
$$

induces a unipotent $\Lambda$-automorphism of $U$ : We repeatedly use the equations ( $\dagger$ ) to expand the elements $\alpha u w_{j} x$ and $v_{i}(\alpha, u) w_{j} x$, for $(\alpha, u) \gtrless p, i \in I(\alpha, u)$ and $j=1, \ldots, t$, in terms of the $K$-basis $x=p_{0} x, p_{1} x, \ldots, p_{l} x$ of $U$. Next we insert these expansions into the equations

$$
\alpha u\left(x+\sum_{j=1}^{t} c_{j} w_{j} x\right)=\sum_{i \in I(\alpha, u)} k_{i}(\alpha, u) v_{i}(\alpha, u)\left(x+\sum_{j=1}^{t} c_{j} w_{j} x\right)
$$

for $(\alpha, u) \varkappa p$, and compare coefficients of the $p_{j} x$ 's so as to obtain a system of linear equations for $c_{1}, \ldots, c_{t}$. This system is homogeneous, say of the form $A(k)\left(c_{1}, \ldots, c_{t}\right)^{T}=0$ for a suitable $K$-matrix $A(k)$, as the 'constant' terms on the two sides of $(\ddagger)$ obviously cancel out. Now $\delta(k)=t-\operatorname{rank} A(k)$, and consequently the dimension of the fibre $\Phi_{p}^{-1} \Phi_{p}(k)$ equals the rank of the matrix $A(k)$.

Example 1. Fixing $l \in \mathbb{N}$, let $\Lambda=K \Gamma / I$ be the algebra based on the quiver $\Gamma$

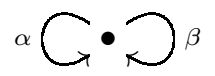

and the ideal $I \subseteq K \Gamma$ generated by all paths of length $l+1$. Moreover, consider the $(l+1)$-term sequence $\mathbb{S}=(S, S, \ldots, S)$, where $S$ is the unique simple left $\Lambda$ module. Given any path $p$ of length $l$, the first of the above remarks permits us to compute $\mathcal{G}-\operatorname{Uni}(p) \cong V_{p} \cong \mathbb{A}^{l(l+1) / 2}$. According to [6, part (3) of Example], we have, moreover, $\mathcal{G}$-Uni $(\mathbb{S}) \cong \mathbb{P}^{1} \times \cdots \times \mathbb{P}^{1} \times \mathbb{A}^{l(l+1) / 2}$ with $l$ factors $\mathbb{P}^{1}$ occurring in the product; in particular, $\mathcal{G}-\operatorname{Uni}(\mathbb{S})$ is irreducible.

First we will see that all of the numbers between 0 and $l-1$ show up as fiber dimensions. For that purpose consider, for instance, the path $p=\alpha^{l-1} \beta$ through $\mathbb{S}$, and for $1 \leq m \leq l$, let $k(m) \in V_{p}$ be a point corresponding to the uniserial module

$$
U(m)=\Lambda /\left(\Lambda\left(\alpha-\beta^{m-1} \alpha\right)+\sum_{0 \leq j \leq l-1} \Lambda \beta \alpha^{j} \beta\right) .
$$

In other words, the detours on $p$ being $(\alpha, 1)$ and $\left(\beta, \alpha^{j} \beta\right)$ for $j \geq 1$, the point $k(m)$ may be chosen to have coordinates $k_{m}(\alpha, 1)=1, k_{i}(\alpha, 1)=0$ for $i \neq m$, and $k_{i}\left(\beta, \alpha^{j} \beta\right)=0$ whenever $j \geq 0$. Using Theorem A and Remark 2 above, it is now easy to compute the dimension of the fibre of $\Phi_{p}$ (or, equivalently, that of the fibre of $\left.\phi_{\mathbb{S}}\right)$ over $U(m)=\Phi_{p}(k(m))$ to be $l-m$.

Next we show that there is no unipotent subgroup $H$ of $\mathrm{GL}_{l+1}$ which acts nontrivially on $\operatorname{Uni}(p)$ by conjugation. This of course entails that there is no conjugation action of a unipotent matrix group $H$ on $\operatorname{Uni}(p)$ such that the orbits of the action coincide with the fibres of the canonical map from $\operatorname{Uni}(p)$ to the isomorphism classes of uniserials with mast $p$. To verify our claim, observe that, in our present setting, $\operatorname{Uni}(p)$ consists of all pairs $(A, B)$ of strictly lower triangular $(l+1) \times(l+1)$-matrices 
representing multiplication by $\alpha$ and $\beta$, with the property that the first column of $B$ consists of the canonical basis vector $e_{2}$, and the second through $l$ th columns of $A$ coincide with $e_{3}$ through $e_{l+1}$, in that order. Now it is readily checked that the only nontrivial unipotent subgroup of $\mathrm{GL}_{l+1}$ which leaves $\operatorname{Uni}(p)$ invariant under conjugation is $\left\{E+c E_{l+1,1} \mid c \in K\right\}$; here $E$ is the identity matrix and the $E_{i j}$ denote the matrix units in $\mathrm{M}_{l+1}(K)$. Since $\operatorname{Uni}(p)$ is made up of strictly lower triangular matrices, this group clearly acts trivially on $\operatorname{Uni}(p)$.

(II) Algebras of finite uniserial type revisited. In [4], the first author settled the following conjecture from [10] affirmatively: If $\Lambda$ has finite uniserial type, then, given any sequence $\mathbb{S}$ of simple $\Lambda$-modules, there is at most one uniserial module (up to isomorphism) having composition series $\mathbb{S}$. We start by indicating a short geometric proof of this fact and then combine it with Theorem A to confirm the remaining conjecture of [10] as well; namely, finite uniserial type of $\Lambda$ forces each nonempty variety $V_{\mathbb{S}}$ to be a full affine space. Our main application, Corollary E, will provide new equivalent characterizations of such algebras which round off the pictures presented in [10] and [4]. All the quantities involved in these descriptions can be computed from quiver and relations of $\Lambda$.

As before, let $\mathbb{S}=(S(0), \ldots, S(l))$ be a sequence of simple left $\Lambda$-modules and set $d=l+1$. Recall that the set $\operatorname{Mod}-\operatorname{Uni}(\mathbb{S}) \subseteq \operatorname{Mod}_{\Lambda}^{d}$, consisting of the points of $\operatorname{Mod}_{\Lambda}^{d}$ that correspond to uniserial modules $U$ with composition series $\mathbb{S}$, is an open subvariety of $\operatorname{Mod}_{\Lambda}^{d}$ which is stable under the $\mathrm{GL}_{d}$-action.

Proposition D [4, Theorem 1]. If $\Lambda$ has finite uniserial type, then, given any finite sequence $\mathbb{S}$ of simple left $\Lambda$-modules, there exists at most one uniserial left $\Lambda$-module with composition series $\mathbb{S}$, up to isomorphism.

Proof. Assume finite uniserial type. Keeping the above notation, we perform an induction on $l$. Clearly, the case $l=0$ is harmless, so suppose that $l \geq 1$ and that $\mathcal{G}-\mathrm{Uni}(\mathbb{S})$ is nonempty; the latter is tantamount to the requirement that the quasi-affine variety Mod-Uni(S) be nonempty. By our induction hypothesis, all of the uniserial modules $U$ with composition series $\mathbb{S}$ have the same radical, up to isomorphism - call it $R$. Consequently, all uniserial modules with composition series $\mathbb{S}$ are extensions of $R$ by $S(0)$. Now it is known that the points in $\operatorname{Mod}_{\Lambda}^{d}$ corresponding to arbitrary extensions of $R$ by $S(0)$ form an irreducible subset $E(R, S(0))$ of $\operatorname{Mod}_{\Lambda}^{d}$ (see, e.g., [3, 6.3]), and since $\operatorname{Mod}-U n i(\mathbb{S})$ is open, the same is true for the intersection $E(R, S(0)) \cap \operatorname{Mod}-U n i(\mathbb{S})$. Investing the fact that $\Lambda$ has finite uniserial type, we infer that this intersection is a finite union of $\mathrm{GL}_{d}$-orbits. All of these orbits being closed in Mod-Uni( $(S)$ by Remark 1 at the end of Section 2, irreducibility implies that there is only one such orbit, which is what we wanted to show.

Again suppose that the primitive idempotent corresponding to the top simple $S(0)$ of $\mathbb{S}$ is $e$ and that $p$ is a path through $\mathbb{S}$. For any point $C \in \mathcal{G}-\mathrm{Uni}(\mathbb{S})$ and any $k \in V_{p}$, we denote by $\delta(C)$ and $\delta(k)$ the $K$-dimensions of the $\Lambda$-endomorphism rings of the uniserial modules $\Lambda e / C$ and $\Phi_{p}(k)$, respectively. Recall that $\mu(\mathbb{S})$ is the multiplicity of $S(0)$ in $\mathbb{S}$; accordingly, we write $\mu(p)$ for $\mu(\mathbb{S})$ whenever $p$ is a path through $\mathbb{S}$. We then obtain the following characterizations of finite uniserial type. On the side, we note that, since polynomials for the varieties $V_{p}$ can be directly obtained from quiver and relations of a split basic algebra, condition (3) is verifiable by means of Gröbner basis methods. The formally strongest condition, (4), demonstrates how restrictive the condition of finite uniserial type is. 
Corollary $\mathbf{E}$ to Theorem A. The following statements are equivalent for $\Lambda=$ $K \Gamma / I$ :

(1) $\Lambda$ has finite uniserial type.

(2) For each finite sequence $\mathbb{S}$ of simple left $\Lambda$-modules and every point $C \in$ $\mathcal{G}-\mathrm{Uni}(\mathbb{S})$, the variety $\mathcal{G}-\mathrm{Uni}(\mathbb{S})$ is either empty or has dimension $\mu(\mathbb{S})-\delta(C)$.

(3) $\Gamma$ has no double arrows, and for each path $p \in K \Gamma$, the affine variety $V_{p}$ is either empty or irreducible of dimension $\mu(p)-\delta(k)$ for some point $k \in V_{p}$.

(4) If $\mathbb{S}$ is the sequence of consecutive composition factors of a uniserial left $\Lambda$ module, then there exists a unique path $p$ through $\mathbb{S}$ and $\mathcal{G}-\operatorname{Uni}(\mathbb{S})=\mathcal{G}-\mathrm{Uni}(p) \cong V_{p}$ is isomorphic to $\mathbb{A}^{\mu(\mathbb{S})-\delta(k)}$ for all points $k \in V_{p}$.

Of course, all statements of the corollary are equivalent to their right-hand analogues.

Proof. (1) $\Longrightarrow(2)$ is a consequence of Proposition D, reproved above, and Theorem A. For $(2) \Longrightarrow(1)$, suppose that $p$ is a path through $\mathbb{S}$ with $\mathcal{G}$-Uni $(p) \neq \varnothing$ and $\mathcal{C}$ an irreducible component of $\mathcal{G}-\operatorname{Uni}(p)$, containing a point $C$ say. Then $\operatorname{dim} \mathcal{C} \leq$ $\mu(\mathbb{S})-\delta(C)$ by $(2)$, and by Theorem $\mathrm{A}$, the fibre of $\Phi_{\mathbb{S}}$ over the isomorphism class of $\Lambda e / C$ is a closed subvariety of $\mathcal{C}$. Moreover, Theorem A shows this fibre to have dimension $\mu(\mathbb{S})-\delta(C)$, making it coincide with $\mathcal{C}$. This establishes finiteness of the number of isomorphism classes of simples with composition series $\mathbb{S}$ as required.

$(1) \Longrightarrow(4)$. Clearly, finite uniserial type excludes the existence of double arrows in $\Gamma$, so that, whenever $\mathcal{G}$-Uni $(\mathbb{S}) \neq \varnothing$, there exists a unique path $p$ through $\mathbb{S}$, and $\mathcal{G}-\operatorname{Uni}(\mathbb{S})=\mathcal{G}-\operatorname{Uni}(p) \cong V_{p}$. The rest follows again from Proposition $\mathrm{D}$ and Theorem A.

The implication (4) $\Longrightarrow(3)$ is obvious, and the reason for $(3) \Longrightarrow(1)$ is analogous to that given for $(2) \Longrightarrow(1)$; note that the irreducibility condition in (3) permits us to cut the dimension hypothesis down to a single point $k \in V_{p}$.

Applying Corollary E to Examples. In case one wishes to decide whether a given algebra $\Lambda=K \Gamma / I$ has finite uniserial type, it is most efficient to start by checking the following strong necessary condition $(\mathrm{N})$ given in [10, Section 3]:

$(\mathrm{N})$ Whenever $\alpha: e \rightarrow e^{\prime}$ is an arrow in $\Gamma$ and $p: e \rightarrow e^{\prime}$ a mast of positive length, $p$ is of the form where $c^{\prime}, c$ are oriented cycles which may be trivial. Given

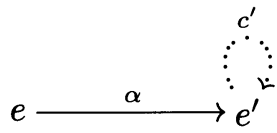

or

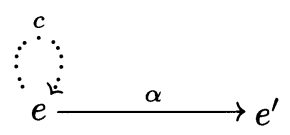

that condition $(\mathrm{N})$ is satisfied, the results of [10, Sections 4 and 5], permit us in many cases to decide 'at a glance' whether, for a given path $p$, there are infinitely many uniserials with mast $p$, up to isomorphism. For the remaining paths, the decision process is most effortlessly carried out with the help of conditions (2), (3) of Corollary E, combined with the concluding remarks of Section 2. We illustrate the procedure with two algebras. For these, answering the question 'finite or infinite uniserial type?' is cumbersome on the basis of previous methods. 
Examples 2. Consider the following quiver $\Gamma$ :

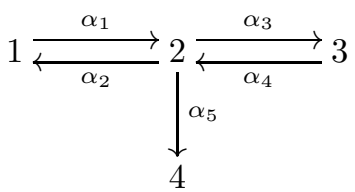

Clearly, each finite dimensional factor algebra $\Lambda$ of $K \Gamma$ satisfies condition (N). In particular, absence of double arrows permits us to identify the variety $\mathcal{G}$-Uni( $(\mathbb{S})$ with $V_{p}$, whenever $p$ is a path through a sequence $\mathbb{S}$ of simple $\Lambda$-modules.

(a) Let $\Lambda=K \Gamma / I$, where $I$ is generated by $\alpha_{2} \alpha_{1} \alpha_{2}, \alpha_{3} \alpha_{4}, \alpha_{2} \alpha_{4}$, and $\alpha_{5} \alpha_{1} \alpha_{2}-$ $\alpha_{5} \alpha_{4} \alpha_{3} \alpha_{1} \alpha_{2}$. Then it is easy to see that the uniserial dimension of $\Lambda$ at $\mathbb{S}^{\prime}$ is $\leq 0$ for all $\mathbb{S}^{\prime} \neq \mathbb{S}=\left(S_{1}, S_{2}, S_{1}, S_{2}, S_{3}, S_{2}, S_{4}\right)$. We will use the computational remarks at the end of Section 2 to check that uniserdim ${ }_{\mathbb{S}} \Lambda=1$, which, in particular, means that $\Lambda$ fails to have finite uniserial type.

The unique path $p$ of length 6 through $\left(e_{1}, e_{2}, e_{1}, e_{2}, e_{3}, e_{2}, e_{4}\right)$ is

$$
p=\alpha_{5} \alpha_{4} \alpha_{3} \alpha_{1} \alpha_{2} \alpha_{1} \text {. }
$$

To compute $V_{p}$, we note that the detours on $p$ are $\left(\alpha_{5}, \alpha_{1}\right),\left(\alpha_{3}, \alpha_{1}\right)$, and $\left(\alpha_{5}, \alpha_{1} \alpha_{2} \alpha_{1}\right)$; they lead to the substitution equations

$$
\alpha_{3} \alpha_{1} \widehat{=} X_{1} \alpha_{3} \alpha_{1} \alpha_{2} \alpha_{1}, \quad \alpha_{5} \alpha_{1} \widehat{=} X_{2} p, \quad \alpha_{5} \alpha_{1} \alpha_{2} \alpha_{1} \widehat{=} X_{3} p .
$$

The relation $\alpha_{5} \alpha_{1} \alpha_{2}-\alpha_{5} \alpha_{4} \alpha_{3} \alpha_{1} \alpha_{2}$ can be supplemented by non-routes on $p$ to a generating set for the left ideal $I e_{1}$, whence insertion of the substitution equations yields $X_{3}=1$ and no conditions impinging on $X_{1}, X_{2}$. Thus, $V_{p}=\mathbb{A}^{2}$. The matrix $A(k)$ of Remark 2 of Section 2 has rank 1 for each $k=\left(k_{1}, k_{2}\right) \in V_{p}$, whence the fibre dimension of $\Phi_{p}^{-1} \Phi_{p}(k)$ is constant on $V_{p}$ and equal to 1 . Thus the uniserial dimension of $\Lambda$ at $\mathbb{S}$ is indeed 1 .

(b) Now let $\Lambda^{\prime}=K \Gamma / I^{\prime}$, where $I^{\prime}$ is generated by $\alpha_{2} \alpha_{1} \alpha_{2}, \alpha_{3} \alpha_{4}, \alpha_{2} \alpha_{4}$, and $\alpha_{5} \alpha_{1}-\alpha_{5} \alpha_{4} \alpha_{3} \alpha_{1}$, and let $\mathbb{S}$ and $p$ be as in part (a). This time, the relations $\alpha_{5} \alpha_{1} \alpha_{2} \alpha_{1}-p$ and $\alpha_{5} \alpha_{1}-\alpha_{5} \alpha_{4} \alpha_{3} \alpha_{1}$ can be supplemented by non-routes on $p$ to a generating set for the left ideal $I^{\prime} e_{1}$, and our substitution equations yield $X_{3}=1$ and $X_{1}=X_{2}$. Thus, $V_{p} \cong \mathbb{A}^{1}$. Again we compute $\operatorname{rank} A(k)=1$ for arbitrary $k \in V_{p}$, which implies that the uniserial dimension of $\Lambda^{\prime}$ at $\mathbb{S}$ equals 0 in this case. The other $V_{q}$ 's can be dealt with in a computation-free manner (see [10]), the conclusion being that $\Lambda^{\prime}$ does have finite uniserial type.

\section{ENDOMORPHISM RINGS OF UNISERIAL MODULES AND AN ALTERNATE ROAD TO THE FIBRE STRUCTURE}

In the following, we will present an alternate approach to the fact that the fibres of the maps $\Phi_{p}$ are full affine spaces, thereby encountering the somewhat surprising fact that all endomorphism rings of uniserial $\Lambda$-modules are commutative (keep in mind that our blanket hypothesis on $\Lambda$ calls for an algebraically closed base field).

Again $p=\alpha_{l} \cdots \alpha_{1}$ will be a fixed path of length $l$ passing through the sequence $(e(0), \ldots, e(l))$ of vertices that accompanies our sequence $\mathbb{S}=(S(0), \ldots, S(l))$ of simples. Moreover, we will continue to identify the preferred primitive idempotents $e_{1}, \ldots, e_{n} \in \Lambda$ with the vertices of $\Gamma$. This time we will work with the isomorphic copy $\operatorname{Uni}(p)$ of $\mathcal{G}-\operatorname{Uni}(p)$, alias $V_{p}$, inside the classical variety $\operatorname{Mod}-\operatorname{Uni}(\mathbb{S})$, as 
introduced at the end of Section 1. Namely,

$$
\operatorname{Uni}(p)=\left\{x=(\alpha(x))_{\alpha \in \Gamma^{*}} \in \operatorname{Mod}-\operatorname{Uni}(\mathbb{S}) \mid \alpha_{i}(x) \mathbf{e}_{i-1}=\mathbf{e}_{i} \text { for } 1 \leq i \leq l\right\},
$$

where $\left(\mathbf{e}_{0}, \ldots, \mathbf{e}_{l}\right)$ is the canonical basis for $K^{l+1}$, and $\Gamma^{*}$ is the union of $\left\{e_{1}, \ldots, e_{n}\right\}$ with the set of arrows of $\Gamma$. Here the components $\alpha(x)$ of a point $x$ are viewed as $(l+1) \times(l+1)$-matrices representing multiplication by the pertinent $\alpha$ relative to the canonical basis; the rows and columns are indexed by $\{0, \ldots, l\}$. In light of the commutative diagram given at the end of Section 1, which links up all of our canonical maps from varieties to module categories, it is harmless to identify $V_{p}$ with the subvariety $\operatorname{Uni}(p)$ of $\operatorname{Mod}-\operatorname{Uni}(\mathbb{S})$, and $\Phi_{p}$ with the restriction $\left.R\right|_{\operatorname{Uni}(p)}$ - we will do this in the sequel. Note that, for any $x \in V_{p}$, there is a uniserial module representing $\Phi_{p}(x)$ having underlying vector space $K^{l+1}$ and composition series $\Lambda \mathbf{e}_{0} \supset \Lambda \mathbf{e}_{1} \supset \cdots \supset \Lambda \mathbf{e}_{l} \supset 0$; this is a trivial consequence of the definitions. Hence all of the matrices $\alpha(x)$, where $\alpha$ runs through the arrows of $\Gamma$, are strictly lower triangular.

We will denote by $\mathbf{d}$ the sequence $\left(d_{1}, \ldots, d_{n}\right)$, where $d_{i}$ is the multiplicity of the simple module $\Lambda e_{i} / J e_{i}$ in $\mathbb{S}$, and by $X_{i}$ the $d_{i}$-dimensional subspace of $X=K^{l+1}$ spanned by those $\mathbf{e}_{j}$ for which $S(j) \cong \Lambda e_{i} / J e_{i}$. Then, clearly, $X=\bigoplus_{1 \leq i \leq n} X_{i}$, and Mod-Uni( $(\mathbb{S})$ is contained in the closed subset $\operatorname{Mod}_{\Lambda}^{\mathbf{d}}$ of $\operatorname{Mod}_{\Lambda}^{l+1}$ which consists of the points $(\alpha(x))_{\alpha \in \Gamma^{*}}$ with $\alpha(x) \in \operatorname{Hom}_{K}\left(X_{s(\alpha)}, X_{t(\alpha)}\right)$, where $s(\alpha)$ and $t(\alpha)$ denote the starting and terminal vertices of $\alpha$, respectively; by this we mean, in particular, that the restriction of $\alpha(x)$ to $\sum_{i \neq s(\alpha)} X_{i}$ is zero. Clearly, $\operatorname{Mod}_{\Lambda}^{\mathrm{d}}$ is stable under conjugation by $\mathrm{GL}(\mathbf{d})=\prod_{1 \leq i \leq n} \mathrm{GL}_{d_{i}}$ if the elements in $\prod_{1 \leq i \leq n} \mathrm{GL}_{d_{i}}$ represent those automorphisms of $X$ which leave the $X_{i}$ invariant, with respect to the canonical basis $\mathbf{e}_{0}, \ldots, \mathbf{e}_{l}$ of $X$. Note that the image of $\operatorname{Mod}_{\Lambda}^{\mathbf{d}}$ under $R$ is just the set of isomorphism classes of $\Lambda$-modules with dimension vector $\mathbf{d}$.

From now on, we will assume that the starting point $e(0)$ of $p$ equals $e_{1}$ in the above listing of the vertices. This entails, in particular, that the first canonical basis vector $\mathbf{e}_{0}$ of $X$ coincides with the first canonical basis vector of $X_{1}$.

We will, moreover, consider two subgroups of the linear group GL(d), the first being $U(\mathbf{d})=\prod_{1 \leq i \leq n} U\left(d_{i}\right)$, where $U\left(d_{i}\right)$ consists of the unipotent lower triangular matrices in $\operatorname{GL}\left(d_{i}\right)$. While the group $U(\mathbf{d})$ acts on $\operatorname{Mod}_{\Lambda}^{\mathbf{d}}$ by conjugation, it also acts on the vector space $X$ by multiplication from the left. The identification of elements of $U(\mathbf{d})$ with $(l+1) \times(l+1)$-matrices is to follow the same pattern as above. Secondly, we consider the stabilizer subgroup $H$ of $\mathbf{e}_{0}$ in $U(\mathbf{d})$ under the latter action; i.e., $H$ is the subgroup of those matrices in $U(\mathbf{d})$ which carry $\mathbf{e}_{0}$ in the 0th column.

Observe that the variety $V_{p} \subseteq \operatorname{Mod}_{\Lambda}^{\mathbf{d}}$ is not stable under conjugation by elements in $U(\mathbf{d})$. But the following subvariety $V_{p}^{\prime}$ of $\operatorname{Mod}_{\Lambda}^{\mathbf{d}}$ containing $V_{p}$ is: Namely, let $V_{p}^{\prime}$ consist of those points $x$, represented by tuples of lower triangular matrices $\alpha(x) \in M_{l+1}(K)$, for which $\alpha_{i}(x)_{i, i-1}=1$. In particular, $V_{p}^{\prime}$ is stable relative to conjugation by elements in $H$.

Lemma 3. Restriction of the $H$-action to $V_{p}$ induces an isomorphism

$$
H \times V_{p} \rightarrow V_{p}^{\prime} .
$$

Proof. The inverse of the morphism $(h, x) \mapsto h . x$ is given by 


$$
y \mapsto\left(g(y), g(y)^{-1} y g(y)\right),
$$

where the columns of $g(y)$ are $\mathbf{e}_{0}, \alpha_{1}(y) \mathbf{e}_{0}, \ldots, \alpha_{l}(y) \alpha_{l-1}(y) \ldots \alpha_{1}(y) \mathbf{e}_{0}$.

We now fix an element $x \in V_{p}$. As before, we let $w_{1}, \ldots, w_{t}$ be the distinct right subpaths of positive length ending in the starting vertex $e$ of $p$, insisting that they be listed in order of increasing length. We write them as successive extensions of one another: Set $u_{1}=w_{1}$, and let $u_{i}$ be the path with $w_{i}=$ $u_{i} w_{i-1}$. Note that we have $\operatorname{dim} X_{1}=d_{1}=t+1$. Clearly, the maps $u_{j}(x)$ induce nilpotent endomorphisms of $X_{1}$, represented by strictly lower triangular matrices, while sending $\sum_{2<i<n} X_{i}$ to zero. The definition of $V_{p}$ moreover guarantees that $w_{t}(x)=u_{t}(x) u_{t-1}(x) \ldots u_{1}(x) \neq 0$ and - our base field $K$ being infinite - we can therefore find a $K$-linear combination $f$ of the maps $u_{1}(x), \ldots, u_{t}(x)$ such that $f^{t}$ does not vanish. Let $A \subseteq U(\mathbf{d})$ be the subgroup consisting of those elements of $U\left(d_{1}\right) \times 1 \times \cdots \times 1$ which commute with $f$. Elementary linear algebra then shows that

- $A$ consists precisely of the linear combinations of positive powers of $f$ added to the identity matrix in $U(\mathbf{d})$ - so, in particular, $A$ is commutative - and

- $A \mathbf{e}_{0}=X_{1}$ inside $K^{l+1}=X_{1} \oplus \cdots \oplus X_{n}$.

Lemma 4. The varieties $H \times A$ and $U(\mathbf{d})$ are isomorphic via the multiplication $\operatorname{map}(h, a) \mapsto h a$.

Proof. We see that $H \cap A=1$, since all nonzero linear combinations of $f, f^{2}, \ldots, f^{t}$ are strictly lower triangular with nonzero first columns. Combined with the second of the preceding observations, this shows that, for each $g \in U(\mathbf{d})$, there exists a unique element $a(g) \in A$ with the property that $g \cdot a(g)$ belongs to $H$, i.e., with $a(g) \mathbf{e}_{0}=g^{-1} \mathbf{e}_{0}$. Next, we note that the assignment $U(\mathbf{d}) \rightarrow A, g \mapsto a(g)$ is a morphism of varieties; indeed, $a(g)=i d+\sum_{i=1}^{t} c_{i} f^{i}$, where the $c_{i}$ are polynomials in the entries of the first column of $g^{-1}$, with coefficients depending only on $f$. Consequently, the inverse to the multiplication map of our claim, namely the map $U(\mathbf{d}) \rightarrow H \times A, g \mapsto\left(g \cdot a(g), a(g)^{-1}\right)$, is a morphism as well.

We are now in a position to prove the following modified version of Theorem A.

Theorem $\mathbf{A}^{\prime}$. Let $U$ be a uniserial module representing the isomorphism class $\Phi_{p}(x)$. Then the following are true:

(1) The group $\operatorname{Aut}_{\Lambda}^{u}(U)$ of unipotent $\Lambda$-automorphisms of $U$ is isomorphic to a subgroup $B$ of $A$. Consequently, all endomorphism algebras of uniserial $\Lambda$-modules are commutative.

(2) The fibre $\Phi_{p}^{-1} \Phi_{p}(x)$ is isomorphic to the commutative unipotent group $A / B$ and, in particular, this fibre is isomorphic to $\mathbb{A}^{m}$ with

$$
m=t-\operatorname{dim} \operatorname{Aut}_{\Lambda}^{u}(U)=t+1-\operatorname{dim} \operatorname{End}_{\Lambda}(U) .
$$

Proof. We may assume that the module $U$ has underlying space $X=K^{l+1}$ and composition series $\Lambda \mathbf{e}_{0} \supset \Lambda \mathbf{e}_{1} \supset \cdots \supset \Lambda \mathbf{e}_{l} \supset 0$. Then we obtain $e_{i} U=X_{i}$ for $1 \leq i \leq n$, and may view $\operatorname{Aut}_{\Lambda}^{u}(U)$ as a subgroup of $U(\mathbf{d})$.

(1) By definition, every element $g \in U(\mathbf{d})$ can be uniquely written in the form $\left(g_{1}, g_{2}\right)$, where $g_{1} \in U\left(d_{1}\right)$ is a unipotent automorphism of $X_{1}$ and $g_{2} \in \prod_{i \geq 2} U\left(d_{i}\right)$ a unipotent automorphism of $\sum_{i \geq 2} X_{i}$. If $g \in \operatorname{Aut}_{\Lambda}^{u}(U)$, then $g$ commutes with the $u_{j}(x)$ and hence with $f$. This means that $\left(g_{1}, i d\right)$ commutes with $f$, i.e., that 
$\left(g_{1}, i d\right) \in A$. Since $\mathbf{e}_{0}$ is a top element of $U$, each $\Lambda$-automorphism $g \in \operatorname{Aut}_{\Lambda}^{u}(U)$ is determined by $g\left(\mathbf{e}_{0}\right)=g_{1}\left(\mathbf{e}_{0}\right)$, and we deduce that the map $\operatorname{Aut}_{\Lambda}^{u}(U) \rightarrow A$, given by $g \mapsto\left(g_{1}, i d\right)$ is a monomorphism of groups. For the final assertion of part (1), we note that each element $f \in \operatorname{End}_{\Lambda}(U)$ is of the form $f=a \cdot i d_{U}+g$, for some scalar $a$ and $g \in \operatorname{Aut}_{\Lambda}^{u}(U)$, and thus infer commutativity of $\operatorname{End}_{\Lambda}(U)$ from that of $\operatorname{Aut}_{\Lambda}^{u}(U)$.

(2) Let $B \subseteq A$ be the image of the group homomorphism under (1). We begin by observing that $\Phi_{p}^{-1} \Phi_{p}(x)=O(x) \cap V_{p}$, where $O(x)$ is the $U(\mathbf{d})$-orbit of $x$ in $V_{p}^{\prime}$. We will show in two steps that this intersection is a geometric quotient of $U(\mathbf{d})$ and will subsequently ascertain that it is indeed isomorphic to $A / B$. Since $A / B$ is a connected unipotent group, Theorem 1 will then guarantee that $O(x) \cap V_{p} \cong$ $A / B \cong \mathbb{A}^{m}$, and our claim will follow from the fact that $\operatorname{dim} A=t$.

On one hand, $O(x)$ is a transitive $U(\mathbf{d})$-space with stabilizer subgroup $\operatorname{Aut}_{\Lambda}^{u}(U)$. Since each fibre of the orbit map is obtainable as the solution set of a system of linear equations, this map is separable, which shows that, as a left $U(\mathbf{d})$-space, $O(x)$ is isomorphic to the homogeneous space $U(\mathbf{d}) / \operatorname{Aut}_{\Lambda}^{u}(U)$. Note that the latter is the geometric quotient relative to the action of $\operatorname{Aut}_{\Lambda}^{u}(U)$ on $U(\mathbf{d})$ by right translations. On the other hand, the isomorphism given in Lemma 3 induces an isomorphism $H \times\left(O(x) \cap V_{p}\right) \cong O(x)$ which is equivariant with respect to left multiplication by elements of $H$. Thus $O(x) \cap V_{p}$ is the geometric quotient of $O(x)$ by the left action of $H$. Combining these facts, [4, Lemma 5.9(b)] now yields that $O(x) \cap V_{p}$ is the geometric quotient of $U(\mathbf{d})$ modulo the action of the product $H \times \operatorname{Aut}_{\Lambda}^{u}(U)$ by left resp. right translations.

To verify that this latter geometric quotient is isomorphic to $A / B$, we will reobtain it along an alternate route. Namely, we will again take two successive quotients of $U(\mathbf{d})$, but this time we will first divide by $H$ and then by $\operatorname{Aut}_{\Lambda}^{u}(U)$. Observe that the isomorphism $H \times A \rightarrow U(\mathbf{d})$ of Lemma 4 is $H \times \operatorname{Aut}_{\Lambda}^{u}(U)$-equivariant: we keep the above action on $U(\mathbf{d})$, let $H$ act on $H \times \operatorname{Aut}_{\Lambda}^{u}(U)$ from the left in the obvious fashion, and define the right action of $\operatorname{Aut}_{\Lambda}^{u}(U)$ on $H \times \operatorname{Aut}_{\Lambda}^{u}(U)$ as follows: $(h, a) \cdot g=\left(h \cdot g a(g), a \cdot a(g)^{-1}\right)$; in checking that this is well-defined, keep in mind that the subgroups $A$ and $\operatorname{Aut}_{\Lambda}^{u}(U)$ of $U(\mathbf{d})$ commute, and note that, for $g \in \operatorname{Aut}_{\Lambda}^{u}(U)$, we have $a(g)=\left(g_{1}, i d\right)^{-1}$ in the notation of part (1). Consequently, the geometric quotient of $U(\mathbf{d})$ by the left action of $H$ is isomorphic to $A$, and the right $\operatorname{Aut}_{\Lambda}^{u}(U)$-action on this partial quotient boils down to right multiplication by elements of $B$. We conclude that the left $A$-space $U(\mathbf{d}) /\left(H \times \operatorname{Aut}_{\Lambda}^{u}(U)\right)$ is isomorphic to the geometric quotient $A / B$ of $A$ modulo the right action of $B$ by translations as required. This completes our argument.

In light of Theorem $\mathrm{A}^{\prime}$, the problem of recognizing the endomorphism algebras of uniserial $\Lambda$-modules among the finite dimensional commutative local $K$-algebras imposes itself. We leave this point to a subsequent investigation.

\section{REFERENCES}

1. M. Auslander, I. Reiten, and S. O. Smalø, Representation theory of Artin algebras, Cambridge University Press, Cambridge, 1995; corrected reprint, 1997. MR 96c:16015 MR 98e:16011

2. K. Bongartz, Gauss-Elimination und der grösste gemeinsame direkte Summand von zwei endlichdimensionalen Moduln, Arch. Math. 53 (1989), 256-258. MR 90g:16025

3. K. Bongartz, On degenerations and extensions of finite dimensional modules, Advances in Math. 121 (1996), 245-287. MR 98e:16012 
4. K. Bongartz, A note on algebras of finite uniserial type, J. Algebra 188 (1997), 513-515. MR 98d:16014

5. K. Bongartz, Some geometric aspects of representation theory, in Algebras and Modules I (I. Reiten, S.O. Smalø, and Ø. Solberg, Eds.), Canad. Math. Soc. Conf. Proc. Series 23 (1998), 1-27. MR 99j:16005

6. K. Bongartz and B. Huisgen-Zimmermann, The geometry of uniserial representations of algebras II. Alternate viewpoints and uniqueness, J. Pure Appl. Algebra (to appear).

7. A. Borel, Linear Algebraic Groups, Second enlarged ed., Springer-Verlag, New York, 1991. MR 92d:20001

8. P. Gabriel, Finite representation type is open, in Representations of Algebras (Ottawa 1974) (V. Dlab and P. Gabriel, eds.), Lecture Notes in Math. 488, Springer-Verlag, Berlin, 1975, pp. 132-155. MR 51:12944

9. B. Huisgen-Zimmermann, The geometry of uniserial representations of finite-dimensional algebras I, J. Pure Appl. Algebra 127 (1998), 39-72. MR 99b:16020

10. B. Huisgen-Zimmermann, The geometry of uniserial representations of finite dimensional algebras. III: Finite uniserial type, Trans. Amer. Math. Soc. 348 (1996), 4775-4812. MR 97f:16027

11. B. Huisgen-Zimmermann and A. Skowronski, The uniserial representation type of tame algebras, in preparation.

12. J.E. Humphreys, Linear Algebraic Groups, Graduate Texts in Mathematics, Vol. 21, Springer-Verlag, New York, 1975. MR 53:633

13. A.D. King, Moduli of representations of finite dimensional algebras, Quart. J. Math. Oxford 45 (1994), 515-530. MR 96a:16009

14. H. Kraft, Geometric methods in representation theory, in Representations of Algebras (Puebla 1980), Lecture Notes in Math. 944, Springer-Verlag, Berlin-New York, 1982, pp. 180-258. MR 84c:14007

15. _ Geometrische Methoden in der Invariantentheorie, Second ed., Vieweg, Braunschweig, 1984. MR 86j:14006

16. L. Le Bruyn, Optimal filtrations on representations of finitedimensional algebras, Trans. Amer. Math. Soc., (to appear); see http://win-www.uia.ac.be/u/lebruyn/PAPERS/optimalnew.dvi.

17. M. Rosenlicht, Questions of rationality for solvable algebraic groups over nonperfect fields, Ann. Mat. Pura Appl., IV Ser. 61 (1963), 97-120. MR 28:2113

FB Mathematik, Universität Gesamthochschule Wuppertal, 42119 Wuppertal, GerMANY

E-mail address: bongartz@math.uni-wuppertal.de

Department of Mathematics, University of California, Santa Barbara, California 93106

E-mail address: birge@math.ucsb.edu 